\title{
ON MORI CONE OF BOTT TOWERS
}

\author{
B. NARASIMHA CHARY
}

Abstract. A Bott tower of height $r$ is a sequence of projective bundles

$$
X_{r} \stackrel{\pi_{r}}{\longrightarrow} X_{r-1} \stackrel{\pi_{r-1}}{\longrightarrow} \cdots \stackrel{\pi_{2}}{\longrightarrow} X_{1}=\mathbb{P}^{1} \stackrel{\pi_{1}}{\longrightarrow} X_{0}=\{p t\},
$$

where $X_{i}=\mathbb{P}\left(\mathcal{O}_{X_{i-1}} \oplus \mathcal{L}_{i-1}\right)$ for a line bundle $\mathcal{L}_{i-1}$ over $X_{i-1}$ for all $1 \leq i \leq r$ and $\mathbb{P}(-)$ denotes the projectivization. These are smooth projective toric varieties and we refer to the top object $X_{r}$ also as a Bott tower. In this article, we study the Mori cone and numerically effective (nef) cone of Bott towers, and we classify Fano, weak Fano and log Fano Bott towers. We prove some vanishing theorems for the cohomology of tangent bundle of Bott towers.

Keywords: Bott towers, Mori cone, primitive relations and toric varieties.

\section{INTRODUCTION}

In [BS58], R. Bott and H. Samelson introduced a family of (smooth differentiable) manifolds which may be viewed as the total spaces of iterated $\mathbb{P}^{1}$-bundles over a point $\{p t\}$, where each $\mathbb{P}^{1}$-bundle is the projectivization of a rank 2 decomposable vector bundle. In [GK94, M. Grossberg and Y. Karshon proved (in complex geometry setting) that these manifolds have a natural action of a compact torus and also obtained some applications to representation theory and symplectic geometry. In [Civ05], Y. Civan proved that these are smooth projective toric varieties. These are called Bott towers, we denote them by $\left\{\left(X_{i}, \pi_{i}\right): 1 \leq i \leq r\right\}$, where

$$
X_{r} \stackrel{\pi_{r}}{\longrightarrow} X_{r-1} \stackrel{\pi_{r-1}}{\longrightarrow} \cdots \stackrel{\pi_{2}}{\longrightarrow} X_{1}=\mathbb{P}^{1} \stackrel{\pi_{1}}{\longrightarrow}\{p t\},
$$

$X_{i}=\mathbb{P}\left(\mathcal{O}_{X_{i-1}} \oplus \mathcal{L}_{i-1}\right)$ for a line bundle $\mathcal{L}_{i-1}$ over $X_{i-1}$ for all $1 \leq i \leq r$ and $r$ is the dimension of $X_{r}$. In [CS11], CMS10] and [Ish12, the authors studied "cohomological rigidity" properties of Bott towers. These also play an important role in algebraic topology and K-theory (see [CR05, [DJ91 and references therein). In this article we refer to $X_{r}$ also as a Bott tower (it is also called Bott manifold).

In this paper we study the geometry of Bott towers in more detail by methods of toric geometry. We work over the field $\mathbb{C}$ of complex numbers. We study the Mori cone of $X_{r}$ and prove that the class of curves corresponding to 'primitive relations $r\left(P_{i}\right)$ ' forms a basis of the real vector space of numerical classes of one-cycles in $X_{r}$ (see Theorem 4.7 and Corollary 4.8). An extremal ray $R$ in the Mori cone is called Mori ray if $R \cdot K_{X_{r}}<0$, where $K_{X_{r}}$ is the canonical divisor in $X_{r}$. We describe extremal rays and Mori rays of the Mori cone of $X_{r}$ (see Theorem 8.1). We characterize the ampleness and numerically effectiveness of line bundles on $X_{r}$ (see Lemma 5.1) and describe the generators of the nef cone of $X_{r}$ (see Theorem 5.7).

Recall that a smooth projective variety $X$ is called Fano (respectively, weak Fano) if its anti-canonical divisor $-K_{X}$ is ample (respectively, nef and big). Following [AS14, we say that

The author is supported by AGIR Pole MSTIC project run by the University of Grenoble Alpes, France. 
a pair $(X, D)$ of a normal projective variety $X$ and an effective $\mathbb{Q}$-divisor $D$ is $\log$ Fano if it is Kawamata log terminal and $-\left(K_{X}+D\right)$ is ample (see Section 7 for more details). We study the Fano, weak Fano and the $\log$ Fano (of the pair $\left(X_{r}, D\right)$ for a suitably chosen divisor $D$ in $X_{r}$ ) properties of the Bott tower $X_{r}$. To describe these results we need some notation. It is known that a Bott tower $\left\{\left(X_{i}, \pi_{i}\right): 1 \leq i \leq r\right\}$ is uniquely determined by an upper triangular matrix $M_{r}$ with integer entries, defined via the first Chern class of the line bundle $\mathcal{L}_{i-1}$ on $X_{i-1}$, where $X_{i}=\mathbb{P}\left(\mathcal{O}_{X_{i-1}} \oplus \mathcal{L}_{i-1}\right)$ for $1 \leq i \leq r$ (see [GK94, Section 2.3], Civ05] and [VT15, Section 7.8]). For more details see Section 2, Let

$$
M_{r}:=\left[\begin{array}{ccccc}
1 & \beta_{12} & \beta_{13} & \ldots & \beta_{1 r} \\
0 & 1 & \beta_{23} & \ldots & \beta_{2 r} \\
0 & 0 & 1 & \ldots & \beta_{3 r} \\
\vdots & \vdots & & \ddots & \vdots \\
0 & \ldots & \ldots & & 1
\end{array}\right]_{r \times r}
$$

where $\beta_{i j}$ 's are integers. Define for $1 \leq i \leq r$,

$$
\eta_{i}^{+}:=\left\{r \geq j>i: \beta_{i j}>0\right\}
$$

and

$$
\eta_{i}^{-}:=\left\{r \geq j>i: \beta_{i j}<0\right\} .
$$

If $\left|\eta_{i}^{+}\right|=1$ (respectively, $\left|\eta_{i}^{+}\right|=2$ ), then let $\eta_{i}^{+}=\{m\}$ (respectively, $\eta_{i}^{+}=\left\{m_{1}, m_{2}\right\}$ ). If $\left|\eta_{i}^{-}\right|=1$ (respectively, $\left|\eta_{i}^{-}\right|=2$ ), then set $\eta_{i}^{-}=\{l\}$ (respectively, $\eta_{i}^{-}=\left\{l_{1}, l_{2}\right\}$ ). The following can be viewed as a condition on $i^{\text {th }}$ row of the matrix $M_{r}$ :

- $N_{i}^{1}$ is the condition that

(i) $\left|\eta_{i}^{+}\right|=0,\left|\eta_{i}^{-}\right| \leq 1$, and if $\left|\eta_{i}^{-}\right|=1$ then $\beta_{i l}=-1$; or

(ii) $\left|\eta_{i}^{-}\right|=0,\left|\eta_{i}^{+}\right| \leq 1$, and if $\left|\eta_{i}^{+}\right|=1$ then $\beta_{i m}=1$ and $\beta_{m k}=0$ for all $k>m$.

- $N_{i}^{2}$ is the condition that

Case 1: Assume that $\left|\eta_{i}^{+}\right|=0$. Then $\left|\eta_{i}^{-}\right| \leq 2$, and if $\left|\eta_{i}^{-}\right|=1$ (respectively, $\left|\eta_{i}^{-}\right|=2$ ) then $\beta_{l i}=-1$ or -2 (respectively, $\beta_{i l_{1}}=-1=\beta_{i l_{2}}$ ).

Case 2: If $\left|\eta_{i}^{-}\right|=1=\left|\eta_{i}^{+}\right|$and $l<m$, then $\beta_{i l}=-1, \beta_{i m}=1$ and $\beta_{m k}=0$ for all $k>m$.

Case 3: Assume that $\left|\eta_{i}^{+}\right|=1$. Then $\beta_{i m}=1$ and either it satisfies

(i) Case 2; or

(ii) $\left|\eta_{i}^{-}\right|=0$ and $\beta_{m k}=0$ for all $k>m$; or

(iii) there exists a unique $r \geq s>m$ such that

$\beta_{m s}-\beta_{i s}=1$ and $\beta_{m k}-\beta_{i k}=0$ for all $k>s$, or

$\beta_{m s}-\beta_{i s}=-1$ and $\beta_{i s}-\beta_{m s}-\beta_{s k}=0$ for all $k>s$.

Definition 1.1. We say $X_{r}$ satisfies condition I (respectively, condition II) if $N_{i}^{1}$ (respectively, $N_{i}^{2}$ ) holds for all $1 \leq i \leq r$.

Note that $N_{i}^{1} \Longrightarrow N_{i}^{2}$ for all $1 \leq i \leq r$. If $X_{r}$ satisfies condition $I$, then it also satisfies conditions $I I$. We prove,

Theorem (see Theorem 6.3).

(1) $X_{r}$ is Fano if and only if it satisfies $I$.

(2) $X_{r}$ is weak Fano if and only if it satisfies II. 
As a consequence we get some vanishing results for the cohomology of tangent bundle of Bott towers and hence local rigidity results. Let $T_{X_{r}}$ denote the tangent bundle of $X_{r}$.

Corollary (see Corollary 6.4 and Corollary 6.5). If $X_{r}$ satisfies $I$, then $H^{i}\left(X_{r}, T_{X_{r}}\right)=0$ for all $i \geq 1$. In particular, $X_{r}$ is locally rigid.

For $1 \leq i \leq r$, we define some constants $k_{i}$ which again depend on the given matrix $M_{r}$ corresponding to the Bott tower $X_{r}$ (for more details see Section 7). We prove,

Theorem (see Theorem 7.1). The pair $\left(X_{r}, D\right)$ is log Fano if and only if $k_{i}<0$ for all $1 \leq i \leq r$.

Remark 1.2. By using the results of this article, in [Cha17b] we give some applications to Bott-Samelson-Demazure-Hansen (BSDH) variety, which can be described also as a iterated projective line bundle, by degeneration of this variety to a Bott tower. Precisely, we study Fano, weak Fano, log Fano properties for BSDH varieties (see also Cha17a). We obtain some vanishing theorems for the cohomology of tangent bundle (and line bundles) on BSDH varieties (see also [CKP15], [CKP] and [CK17]). We also recover the results in [PK16].

The paper is organized as follows: In Section 2, we discuss preliminaries on Bott towers and toric varieties. In Section 3, we discuss the Picard group of the Bott tower and compute the relative tangent bundle. Section 4 contains detailed study of primitive collections and primitive relations of the Bott tower and we also describe the Mori cone. In Section 5 we describe ample and nef line bundles on the Bott tower, and we find the generators of the nef cone. In Section 6 and 7, we study Fano, weak Fano and log Fan properties for Bott towers. We also see some vanishing results. In Section 8, we describe extremal rays and Mori rays for the Bott tower.

\section{Preliminaries}

In this section we recall toric varieties (see [CLS11]) and Bott towers (see [Civ05] and [VT15]). We work throughout the article over the field $\mathbb{C}$ of complex numbers. We expect that the proofs work for algebraically closed fields of arbitrary characteristic, but did not find appropriate references in that generality.

2.1. Toric varieties. We briefly recall the structure of toric varieties from [CLS11] (see also [Ful93 and Oda88]).

Definition 2.1. A normal variety $X$ is called a toric variety (of dimension $n$ ) if it contains an $n$-dimensional torus $T$ (i.e. $\left.T=\left(\mathbb{C}^{*}\right)^{n}\right)$ as a Zariski open subset such that the action of the torus on itself by multiplication extends to an action of the torus on $X$.

Toric varieties are completely described by the combinatorics of the corresponding fans. We briefly recall here, let $N$ be the lattice of one-parameter subgroups of $T$ and let $M$ be the lattice of characters of $T$. Let $M_{\mathbb{R}}:=M \otimes \mathbb{R}$ and $N_{\mathbb{R}}:=N \otimes \mathbb{R}$. Then we have a natural bilinear pairing

$$
\langle-,-\rangle: M_{\mathbb{R}} \times N_{\mathbb{R}} \rightarrow \mathbb{R} .
$$

A fan $\Sigma$ in $N_{\mathbb{R}}$ is a collection of convex polyhedral cones that is closed under intersections and cone faces. Let $\check{\sigma}$ be the dual cone of $\sigma \in \Sigma$ in $M_{\mathbb{R}}$. For $\sigma \in \Sigma$, the semigroup algebra $\mathbb{C}[\check{\sigma} \cap M]$ is a normal domain and finitely generated $\mathbb{C}$-algebra. Then the scheme $\operatorname{Spec}(\mathbb{C}[\check{\sigma} \cap M])$ is called 
the affine toric variety corresponding to $\sigma$. For a given fan $\Sigma$, we can define a toric variety $X_{\Sigma}$ by gluing the affine toric varieties $\operatorname{Spec}(\mathbb{C}[\check{\sigma} \cap M])$ as $\sigma$ varies in $\Sigma$. For all $1 \leq s \leq n$,

$$
\Sigma(s):=\{\sigma \in \Sigma: \operatorname{dim}(\sigma)=s\}
$$

For each $\rho \in \Sigma(1)$, we denote $u_{\rho}$, the generator of $\rho \cap N$. For $\sigma \in \Sigma$,

$$
\sigma(1):=\Sigma(1) \cap \sigma .
$$

There is a bijective correspondence between the cones in $\Sigma$ and the $T$-orbits in $X_{\Sigma}$. For each $\sigma \in \Sigma$, the dimension $\operatorname{dim}(O(\sigma))$ of the T-orbit $O(\sigma)$ corresponding to $\sigma$ is $n-\operatorname{dim}(\sigma)$. Let $\tau, \sigma \in \Sigma$, then $\tau$ is a face of $\sigma$ if and only if $O(\sigma) \subset \overline{O(\tau)}$, where $\overline{O(\sigma)}$ is the closure of $T$-orbit $O(\sigma)$. We denote $V(\sigma)=\overline{O(\sigma)}$ and it is a toric variety with the corresponding fan being $\operatorname{Star}(\sigma)$, the star of $\sigma$ which is the set of cones in $\Sigma$ which have $\sigma$ as a face. Let $D_{\rho}=\overline{O(\rho)}$ be the torus-invariant prime divisor in $X_{\Sigma}$ corresponding to $\rho \in \Sigma(1)$. The group TDiv $\left(X_{\Sigma}\right)$ of $T$-invariant divisors in $X_{\Sigma}$ is given by

$$
\operatorname{TDiv}\left(X_{\Sigma}\right)=\bigoplus_{\rho \in \Sigma(1)} \mathbb{Z} D_{\rho}
$$

For each $m \in M$, the character $\chi^{m}$ of $T$ is a rational function on $X_{\Sigma}$ and the corresponding divisor is given by

$$
\operatorname{div}\left(\chi^{m}\right)=\sum_{\rho \in \Sigma(1)}\left\langle m, u_{\rho}\right\rangle D_{\rho}
$$

2.2. Bott towers. In this section we recall some basic definitions and results on Bott towers. Let $\mathcal{L}_{0}$ be a trivial line bundle over a single point $X_{0}:=\{p t\}$, and let $X_{1}:=\mathbb{P}\left(\mathcal{O}_{X_{0}} \oplus \mathcal{L}_{0}\right)$, where $\mathbb{P}(-)$ denotes the projectivization. Let $\mathcal{L}_{1}$ be a line bundle on $X_{1}$, then define $X_{2}:=$ $\mathbb{P}\left(\mathcal{O}_{X_{1}} \oplus \mathcal{L}_{1}\right)$, which is a $\mathbb{P}^{1}$-bundle over $X_{1}$. Repeat this process $r$-times, so that each $X_{i}$ is a $\mathbb{P}^{1}$-bundle over $X_{i-1}$ for $1 \leq i \leq r$. We get the following:

$$
\begin{gathered}
X_{r}=\mathbb{P}\left(\mathcal{O}_{X_{r-1}} \oplus \mathcal{L}_{r-1}\right) \\
\downarrow_{\pi_{r}}=\mathbb{P}\left(\mathcal{O}_{X_{r-2}} \oplus \mathcal{L}_{r-2}\right) \\
\downarrow_{\pi_{r-1}} \\
\vdots \\
\downarrow_{\pi_{2}} \\
X_{1}=\mathbb{P}\left(\mathcal{O}_{X_{0}} \oplus \mathcal{L}_{0}\right) \\
\downarrow_{\pi_{1}} \\
X_{0}=\{p t\}
\end{gathered}
$$

For each $1 \leq i \leq r, X_{i}$ is a smooth projective toric variety (see Civ05, Theorem 22]). Consider the points $[1: 0]$ and $[0: 1]$ in $\mathbb{P}^{1}$, we call them the south pole and the north pole respectively. The zero section of $\mathcal{L}_{i-1}$ gives a section $s_{i}^{0}: X_{i-1} \longrightarrow X_{i}$, the south pole section; similarly, the north pole section $s_{i}^{1}: X_{i-1} \longrightarrow X_{i}$ by letting the first coordinate in $\mathbb{P}\left(\mathcal{O}_{X_{i-1}} \oplus \mathcal{L}_{i-1}\right)$ to vanish. 
Let $1 \leq i \leq r$. Since $\pi_{i}: X_{i} \longrightarrow X_{i-1}$ is a projective bundle, by a standard result on the cohomology ring of projective bundles we have the following (see [Har77, Page 429] for instance, and also [Mil16, Proposition 10.1]):

Theorem 2.2. The cohomology ring $H^{*}\left(X_{i}, \mathbb{Z}\right)$ of $X_{i}$ is a free module over $H^{*}\left(X_{i-1}, \mathbb{Z}\right)$ on generators 1 and $u_{i}$, which have degree 0 and 2 respectively, that is

$$
H^{*}\left(X_{i}, \mathbb{Z}\right)=H^{*}\left(X_{i-1}, \mathbb{Z}\right) 1 \oplus H^{*}\left(X_{i-1}, \mathbb{Z}\right) u_{i}
$$

The ring structure is determined by the single relation

$$
u_{i}^{2}=c_{1}\left(\mathcal{L}_{i-1}\right) u_{i}
$$

where $c_{1}(-)$ denotes the first Chern class and the restriction of $u_{i}$ to the fiber $\mathbb{P}^{1} \subset X_{i}$ is the first Chern class of the canonical line bundle over $\mathbb{P}^{1}$. Hence we have

$$
H^{*}\left(X_{i}, \mathbb{Z}\right)=H^{*}\left(X_{i-1}, \mathbb{Z}\right)\left[u_{i}\right] / J_{i},
$$

where $J_{i}$ is the ideal generated by $u_{i}^{2}-c_{1}\left(\mathcal{L}_{i-1}\right) u_{i}$.

Consider the exponential sequence (see [Har77, Page 446]):

$$
0 \longrightarrow \mathbb{Z} \longrightarrow \mathcal{O}_{X_{i-1}} \longrightarrow \mathcal{O}_{X_{i-1}}^{*} \longrightarrow 0
$$

Then we get the following exact sequence:

$$
\begin{aligned}
0 \longrightarrow H^{1}\left(X_{i-1}, \mathbb{Z}\right) \longrightarrow H^{1}\left(X_{i-1}, \mathcal{O}_{X_{i-1}}\right) \longrightarrow H^{1}\left(X_{i-1}, \mathcal{O}_{X_{i-1}^{*}}^{*}\right) \stackrel{c_{1}(-)}{\longrightarrow} H^{2}\left(X_{i-1}, \mathbb{Z}\right) \longrightarrow \\
H^{2}\left(X_{i-1}, \mathcal{O}_{X_{i-1}}\right) \longrightarrow \cdots
\end{aligned}
$$

Since $X_{i-1}$ is toric, we have $H^{j}\left(X_{i-1}, \mathcal{O}_{X_{i-1}}\right)=0$ for all $j>0$ (see Oda88, Corollary 2.8]). As $H^{1}\left(X_{i-1}, \mathcal{O}_{X_{i-1}}^{*}\right)=\operatorname{Pic}\left(X_{i-1}\right)$, we get $c_{1}(-): \operatorname{Pic}\left(X_{i-1}\right) \stackrel{\sim}{\longrightarrow} H^{2}\left(X_{i-1}, \mathbb{Z}\right)$. Then we have the following:

Theorem 2.3. Each line bundle $\mathcal{L}_{i-1}$ on $X_{i-1}$ is determined (up to an algebraic isomorphism) by its first Chern class, which can be written as a linear combination

$$
c_{1}\left(\mathcal{L}_{i-1}\right)=-\sum_{k=1}^{i-1} \beta_{k i} u_{k} \in H^{2}\left(X_{i-1}, \mathbb{Z}\right),
$$

where $\beta_{i k}$ 's are integers for $1 \leq k \leq i-1$.

Then by Theorem 2.2 and 2.3 , by iteration, we get the following:

Corollary 2.4. We have

$$
H^{*}\left(X_{r}, \mathbb{Z}\right)=\mathbb{Z}\left[u_{1}, \ldots, u_{r}\right] / J
$$

where $J$ is the ideal generated by $\left\{u_{j}^{2}+\sum_{i<j} \beta_{i j} u_{i} u_{j}: 1 \leq j \leq r\right\}$ and the integers $\beta_{i j}$ 's are as in Theorem 2.3.

Write $\left\{\beta_{i j}: 1 \leq i<j \leq r\right\}$, the collection of $r(r-1) / 2$ integers, as an upper triangular $r \times r$ matrix

$$
M_{r}:=\left[\begin{array}{ccccc}
1 & \beta_{12} & \beta_{13} & \ldots & \beta_{1 r} \\
0 & 1 & \beta_{23} & \ldots & \beta_{2 r} \\
0 & 0 & 1 & \ldots & \beta_{3 r} \\
\vdots & \vdots & & \ddots & \vdots \\
0 & \ldots & \ldots & & 1
\end{array}\right]_{r \times r}
$$


Then we get the following result (see for instance [GK94, Lemma 2.15] and also [Civ05, Section $3])$.

Corollary 2.5. There is a bijective correspondence between $\{$ Bott towers of height $r\}$ and $\{r \times r$ upper triangular matrices with integer entries as in (2.1)\}.

Two Bott towers $\left\{\left(X_{i}, \pi_{i}\right): 1 \leq i \leq r\right\}$ and $\left\{\left(X_{i}^{\prime}, \pi_{i}^{\prime}\right): 1 \leq i \leq r\right\}$ are isomorphic if there exists a collection of isomorphisms $\left\{\phi_{i}: X_{i} \rightarrow X_{i}^{\prime}: 1 \leq i \leq r\right\}$ such that the following diagram is commutative:

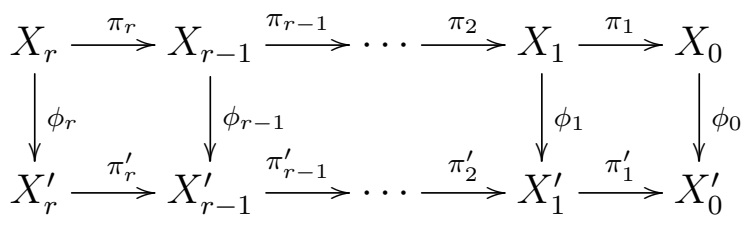

2.2.1. Toric structure on Bott tower. Let $\left\{e_{1}^{+}, \ldots, e_{r}^{+}\right\}$be the standard basis of the lattice $\mathbb{Z}^{r}$. Define, for all $i \in\{1, \ldots, r\}$,

$$
e_{i}^{-}:=-e_{i}^{+}-\sum_{j>i} \beta_{i j} e_{j}^{+}
$$

where $\beta_{i j}$ 's are integers as above. Then we have the following theorem (see Civ05, Section 3 and Theorem 22] and for algebraic topology setting see [VT15, Theorem 7.8.7]):

Theorem 2.6. The Bott tower $\left\{\left(X_{i}, \pi_{i}\right): 1 \leq i \leq r\right\}$ corresponding to a matrix $M_{r}$ as in (2.1) is isomorphic to $\left\{\left(X_{\Sigma_{i}}, \pi_{\Sigma_{i}}\right): 1 \leq i \leq r\right\}$, the collection of smooth projective toric varieties corresponding to the fan $\Sigma_{i}$ with the $2^{i}$ maximal cones generated by the set of vectors

$$
\left\{e_{j}^{\epsilon}: 1 \leq j \leq i \text { and } \epsilon \in\{+,-\}\right\} \text {, }
$$

and where $\pi_{\Sigma_{i}}: X_{\Sigma_{i}} \rightarrow X_{\Sigma_{i-1}}$ is the toric morphism induced by the projection $\overline{\pi_{\Sigma_{i}}}: \mathbb{Z}^{i} \rightarrow \mathbb{Z}^{i-1}$ for all $1 \leq i \leq r$.

Note that by Theorem 2.6, $\Sigma_{i}$ has $2 i$ one-dimensional cones generated by the vectors

$$
\left\{e_{j}^{+}, e_{j}^{-}: 1 \leq j \leq i\right\},
$$

and by 2.2 , we can see that the divisors $D_{\rho_{j}^{+}}$corresponding to $e_{j}^{+}$for $1 \leq j \leq i$ form a basis of the Picard group of $X_{i}$ (see Section 3 for more details).

\section{On PicARd Group of A Bott tower}

Now we describe a basis of the Picard group $\operatorname{Pic}\left(X_{r}\right)$ of $X_{r}$. Let $\epsilon \in\{+,-\}$ and for $1 \leq i \leq r$, let $\rho_{i}^{\epsilon}$ be the one-dimensional cone generated by $e_{i}^{\epsilon}$. For all $1 \leq i \leq r$, we define $D_{\rho_{i}^{\epsilon}}$ to be the toric divisor corresponding to the one-dimensional cone $\rho_{i}^{\epsilon}$. We prove,

Lemma 3.1. The set $\left\{D_{\rho_{i}^{\epsilon}}: 1 \leq i \leq r\right.$ and $\left.\epsilon \in\{+,-\}\right\}$ forms a basis of Pic $\left(X_{r}\right)$.

Proof. By Theorem 2.6, using the description of the one-dimensional cones we have the following decomposition of $\Sigma(1)$ :

$$
\Sigma(1)=\left\{\rho_{i}^{+}: 1 \leq i \leq r\right\} \cup\left\{\rho_{i}^{-}: 1 \leq i \leq r\right\}
$$


Again by Theorem 2.6. $\left\{D_{\rho_{i}^{+}}: 1 \leq i \leq r\right\}$ forms a basis of the Picard group Pic $\left(X_{r}\right)$ of $X_{r}$. Since

$$
0 \sim \operatorname{div}\left(\chi^{e_{i}^{+}}\right)=\sum_{\rho \in \Sigma(1)}\left\langle u_{\rho}, e_{i}^{+}\right\rangle D_{\rho}
$$

by 2.2 we can see that $\left\{D_{\rho_{i}^{-}}: 1 \leq i \leq r\right\}$ also forms a basis of $\operatorname{Pic}\left(X_{r}\right)$. In general, let $\sigma \in \Sigma$ be the maximal cone generated by $\left\{e_{i}^{\epsilon}: 1 \leq i \leq r\right\}$. Take the torus-fixed point $x^{\epsilon}$ in $X_{r}$ corresponding to the maximal cone $\sigma$. Let $U$ be the torus-invariant open affine neighbourhood of $x^{\epsilon}$ in $X_{r}$. Then $U$ is an affine space of dimension $r$; in particular, $\operatorname{Pic}(U)=0$. Therefore, we get

$$
X_{r} \backslash U=\cup_{i=1}^{r} D_{\rho_{i}^{\epsilon}}
$$

and $\operatorname{Pic}\left(X_{r}\right)$ is generated by $\left\{D_{\rho_{i}^{\epsilon}}: 1 \leq i \leq r\right\}$ (see Har70, Chapter II, Proposition 3.1, page 66]). Since $\left\{D_{\rho_{i}^{\epsilon}}: 1 \leq i \leq r\right\}$ is linearly independent and the rank of $\operatorname{Pic}\left(X_{r}\right)$ is $r$, this set $\left\{D_{\rho_{i}^{\epsilon}}: 1 \leq i \leq r\right\}$ forms a basis of $\operatorname{Pic}\left(X_{r}\right)$.

By Lemma 3.1, the set $\left\{D_{\rho_{i}^{+}}: 1 \leq i \leq r\right\}$ forms a basis of $\operatorname{Pic}\left(X_{r}\right)$. Now we express for each $1 \leq i \leq r, D_{\rho_{i}^{-}}$in terms of $D_{\rho_{j}^{+}}$'s $(1 \leq j \leq r)$. Let $1 \leq i \leq r$, define $h_{i}^{i-1}:=-\beta_{i(i-1)}$ and

$$
h_{i}^{j}:= \begin{cases}0 & \text { for } j>i . \\ 1 & \text { for } j=i \\ -\sum_{k=j}^{i-1} \beta_{i k}\left(h_{k}^{j}\right) & \text { for } j<i\end{cases}
$$

Then we prove,

Lemma 3.2. Let $1 \leq i \leq r$. The coefficient of $D_{\rho_{j}^{+}}$in $D_{\rho_{i}^{-}}$is $h_{i}^{j}$.

Proof. Proof is by induction on $i$ and by using

$$
0 \sim \operatorname{div}\left(\chi^{e_{i}^{+}}\right)=\sum_{\rho \in \Sigma(1)}\left\langle u_{\rho}, e_{i}^{+}\right\rangle D_{\rho} .
$$

Recall the equation 2.2 ,

$$
e_{i}^{-}=-e_{i}^{+}-\sum_{j>i} \beta_{i j} e_{j}^{+} \text {for all } 1 \leq i \leq r .
$$

If $i=1$, by 3.2 , we see

Then we have

$$
0 \sim \operatorname{div}\left(\chi^{e_{1}^{+}}\right)=D_{\rho_{1}^{+}}-D_{\rho_{1}^{-}}
$$

$$
D_{\rho_{1}^{-}} \sim D_{\rho_{1}^{+}}
$$

If $i=2$, by 3.2 and 2.2 , we see

$$
0 \sim \operatorname{div}\left(\chi^{e_{2}^{+}}\right)=D_{\rho_{2}^{+}}-D_{\rho_{2}^{-}}-\beta_{21} D_{\rho_{1}^{-}} .
$$

By $(3.3)$, we get

$$
D_{\rho_{2}^{-}} \sim D_{\rho_{2}^{+}}-\beta_{21} D_{\rho_{1}^{+}}=h_{2}^{2} D_{\rho_{2}^{+}}+h_{2}^{1} D_{\rho_{1}^{+}} .
$$

By induction assume that

$$
D_{\rho_{k}^{-}} \sim \sum_{j=1}^{r} h_{k}^{j} D_{\rho_{j}^{+}} \text {for all } k<i
$$


Again by 3.2 and 2.2 , we see

$$
0 \sim \operatorname{div}\left(\chi^{e_{i}^{+}}\right)=D_{\rho_{i}^{+}}-D_{\rho_{i}^{-}}-\sum_{k<i} \beta_{i k} D_{\rho_{k}^{-}} .
$$

Then

$$
D_{\rho_{i}^{-}} \sim D_{\rho_{i}^{+}}-\sum_{k<i} \beta_{i k} D_{\rho_{k}^{-}}
$$

Hence

$$
D_{\rho_{i}^{-}} \sim D_{\rho_{i}^{+}}-\sum_{k<i} \beta_{i k}\left(\sum_{j=1}^{r} h_{k}^{j} D_{\rho_{j}^{+}}\right) .
$$

Since $h_{k}^{j}=0$ for $k<j$, we get

$$
D_{\rho_{i}^{-}} \sim D_{\rho_{i}^{+}}-\sum_{k<i} \beta_{i k}\left(\sum_{j=1}^{i-1} h_{k}^{j} D_{\rho_{j}^{+}}\right)
$$

Then

$$
D_{\rho_{i}^{-}} \sim D_{\rho_{i}^{+}}+\sum_{j=1}^{i-1}\left(-\sum_{k=j}^{i-1} \beta_{i k} h_{k}^{j}\right) D_{\rho_{j}^{+}}
$$

Therefore, we conclude that $D_{\rho_{i}^{-}} \sim D_{\rho_{i}^{+}}+\sum_{j=1}^{i-1} h_{i}^{j} D_{\rho_{j}^{+}}$. This completes the proof of the lemma.

Let $\epsilon \in\{+,-\}$. Define $\Sigma(1)^{\epsilon}:=\left\{\rho_{i}^{\epsilon}: 1 \leq i \leq r\right\}$. Then

$$
D=\sum_{\rho \in \Sigma(1)} a_{\rho} D_{\rho}=\sum_{\rho \in \Sigma(1)^{+}} a_{\rho} D_{\rho}+\sum_{\rho \in \Sigma(1)^{-}} a_{\rho} D_{\rho} .
$$

For $1 \leq i \leq r$, let $g_{i}:=a_{\rho_{i}^{+}}+\sum_{j=i}^{r} a_{\rho_{j}^{-}} h_{j}^{i}$. Then we have

Corollary 3.3. $D=\sum_{\rho \in \Sigma(1)} a_{\rho} D_{\rho} \sim \sum_{i=1}^{r} g_{i} D_{\rho_{i}^{+}}$.

Proof. We have $D=\sum_{\rho \in \Sigma(1)} a_{\rho} D_{\rho}=\sum_{i=1}^{r} a_{\rho_{i}^{+}} D_{\rho_{i}^{+}}+\sum_{i=1}^{r} a_{\rho_{i}^{-}} D_{\rho_{i}^{-}}$. By Lemma 3.2, we can see that $\sum_{i=1}^{r} a_{\rho_{i}^{-}} D_{\rho_{i}^{-}} \sim \sum_{i=1}^{r} a_{\rho_{i}^{-}}\left(\sum_{j=1}^{i} h_{i}^{j} D_{\rho_{j}^{+}}\right)$. Then $\sum_{i=1}^{r} a_{\rho_{i}^{-}} D_{\rho_{i}^{-}} \sim \sum_{i=1}^{r}\left(\sum_{j=i}^{r} a_{\rho_{j}^{-}} h_{j}^{i}\right) D_{\rho_{j}^{+}}$. Hence we have $D \sim \sum_{i=1}^{r}\left(a_{\rho_{i}^{+}}+\sum_{j=i}^{r} a_{\rho_{j}^{-}} h_{j}^{i}\right) D_{\rho_{i}^{+}}$. Thus, $D \sim \sum_{i=1}^{r} g_{i} D_{\rho_{i}^{+}}$and this completes the proof.

Remark 3.4. By Corollary 3.3, we see some vanishing results of the cohomology of line bundles on $B S D H$ varieties in Cha17b.

Let $1 \leq i \leq r$. We prove the following.

Lemma 3.5. The relative tangent bundle $T_{\pi_{i}}$ of $\pi_{i}: X_{i} \rightarrow X_{i-1}$ is given by

$$
T_{\pi_{i}} \simeq \mathcal{O}_{X_{i}}\left(D_{\rho_{i}^{+}}+D_{\rho_{i}^{-}}\right) \simeq \mathcal{O}_{X_{i}}\left(\sum_{j=1}^{i-1} \beta_{i j} D_{\rho_{j}^{-}}+2 D_{\rho_{i}^{-}}\right)
$$


Proof. By definition of Bott tower, $\pi_{i}$ is a $\mathbb{P}^{1}$-fibration. Then the relative canonical bundle $K_{\pi_{i}}$ is given by

$$
K_{\pi_{i}}=\mathcal{O}_{X_{i}}\left(K_{X_{i}}\right) \otimes \pi_{i}^{*}\left(\mathcal{O}_{X_{i-1}}\left(-K_{X_{i-1}}\right)\right)
$$

(see [Kle80, Corollary 24, page 56]). By [CLS11, Theorem 8.2.3] (see also [Ful93, Page 74]), we have

$$
K_{X_{\Sigma}}=-\sum_{\rho \in \Sigma(1)} D_{\rho}
$$

Then

$$
K_{\pi_{i}}=\mathcal{O}_{X_{i}}\left(-\sum_{\rho \in \Sigma(1)} D_{\rho}\right) \otimes \pi_{i}^{*}\left(\mathcal{O}_{X_{i-1}}\left(\sum_{\rho^{\prime} \in \Sigma^{\prime}(1)} D_{\rho^{\prime}}\right)\right)
$$

where $\Sigma^{\prime}$ is the fan of $X_{i-1}$. Since $X_{i-1}$ smooth, any divisor of the form $D=\sum_{\rho^{\prime} \in \Sigma^{\prime}(1)} a_{\rho^{\prime}} D_{\rho^{\prime}}$ with $a_{\rho^{\prime}} \in \mathbb{Z}$, in $X_{i-1}$ is Cartier. Hence the pullback $\pi_{i}^{*}(D)$ is defined and given by

$$
\pi_{i}^{*}(D)=\pi_{i}^{*}\left(\sum_{\rho^{\prime} \in \Sigma^{\prime}(1)} a_{\rho^{\prime}} D_{\rho^{\prime}}\right)=\sum_{\rho \in \Sigma(1)}-\varphi_{D}\left(\bar{\pi}_{i}\left(u_{\rho}\right)\right) D_{\rho}
$$

where $\varphi_{D}$ is the support function corresponding to the divisor $D$ (see [CLS11, Theorem 4.2.12] for the correspondence between support functions and Cartier divisors). Since the lattice map $\bar{\pi}_{i}: \mathbb{Z}^{i} \rightarrow \mathbb{Z}^{i-1}$ is the projection onto the first $i-1$ factors (see page 6 ), by definition of $u_{\rho}$ and $e_{j}^{-}($see 2.2$)$ ), for $\epsilon \in\{+,-\}$ we have

$$
\bar{\pi}_{i}\left(u_{\rho_{j}^{\epsilon}}\right)= \begin{cases}u_{\rho_{j}^{\prime} \epsilon} & \text { if } 1 \leq j \leq i-1 . \\ 0 & \text { if } j=i\end{cases}
$$

Hence

$$
-\varphi_{D}\left(\bar{\pi}_{i}\left(u_{\rho_{j}^{\epsilon}}\right)\right)= \begin{cases}a_{\rho_{j}^{\prime} \epsilon} & \text { if } 1 \leq j \leq i-1 . \\ 0 & \text { if } j=i\end{cases}
$$

Thus we have,

$$
\pi_{i}^{*}\left(\sum_{\rho^{\prime} \in \Sigma^{\prime}(1)} D_{\rho^{\prime}}\right)=\sum_{\rho \in \Sigma(1) \backslash\left\{\rho_{i}^{+}, \rho_{i}^{-}\right\}} D_{\rho} .
$$

Therefore, we see that

$$
K_{\pi_{i}}=\mathcal{O}_{X_{i}}\left(-D_{\rho_{i}^{+}}-D_{\rho_{i}^{-}}\right)
$$

By (2.2), we note that

$$
0 \sim \operatorname{div}\left(\chi^{e_{i}^{+}}\right)=D_{\rho_{i}^{+}}-D_{\rho_{i}^{-}}-\sum_{j=1}^{i-1} \beta_{i j} D_{\rho_{j}^{-}} .
$$

Since $\check{K}_{\pi_{i}}=\operatorname{det} T_{\pi_{i}}$, we get $\check{K}_{\pi_{i}}=T_{\pi_{i}}$ as $\pi_{i}$ is a $\mathbb{P}^{1}$-fibration. Therefore, the result follows from (3.4) and (3.5).

Remark 3.6. By Lemma 3.2, the relative tangent bundle $T_{\pi_{i}}$ can be expressed in terms of $D_{\rho_{i}^{+}}$ $(1 \leq i \leq r)$.

The following is well known and proved here for completeness. 
Lemma 3.7. Let $X$ and $Y$ be smooth varieties. Let $f: X \longrightarrow Y$ be a fibration with a section $\sigma$ and denote by $\sigma(Y)$ its image in $X$. Then the restriction of the relative tangent bundle $T_{f}$ to $\sigma(Y)$ is isomorphic to the normal bundle $\mathcal{N}_{\sigma(Y) / X}$ of $\sigma(Y)$ in $X$.

Proof. Consider the normal bundle short exact sequence

$$
\left.0 \longrightarrow T_{\sigma(Y)} \longrightarrow T_{X}\right|_{\sigma(Y)} \longrightarrow \mathcal{N}_{\sigma(Y) / X} \longrightarrow 0
$$

where $T_{\sigma(Y)}$ and $T_{X}$ are the tangent bundles of $\sigma(Y)$ and $X$ respectively. Also consider the following short exact sequence

$$
0 \longrightarrow T_{f} \longrightarrow T_{X} \longrightarrow f^{*} T_{Y} \longrightarrow 0 .
$$

By restricting (3.7) to $\sigma(Y)$, since $\sigma$ is a section of $f$, we get the following short exact sequence

$$
\left.\left.0 \longrightarrow T_{f}\right|_{\sigma(Y)} \longrightarrow T_{X}\right|_{\sigma(Y)} \longrightarrow T_{\sigma(Y)} \longrightarrow 0 \text {. }
$$

By using (3.6) and (3.8), we see $\left.T_{f}\right|_{\sigma(Y)}$ is isomorphic to $\mathcal{N}_{\sigma(Y) / X}$. This completes the proof.

We prove,

Lemma 3.8. Let $1 \leq i \leq r$. The normal bundle $\mathcal{N}_{X_{i} / X_{i-1}}$ of $X_{i-1}$ in $X_{i}$ is $\check{\mathscr{L}}_{i-1}$, where $\mathscr{L}_{i-1}$ is as in the definition of Bott tower and $\check{\mathscr{L}}_{i-1}$ is denotes the dual of $\mathscr{L}_{i-1}$.

Proof. Fix $1 \leq i \leq r$ and let $\mathscr{L}:=\mathscr{L}_{i-1}$. Recall that $\mathbb{P}(\mathscr{E})$ is by definition $\operatorname{Proj}(S(\mathscr{E})), S(\mathscr{E})$ is symmetric algebra of $\mathscr{E}=\mathcal{O}_{X_{i-1}} \oplus \mathscr{L}$ (see [Har77, Page 162]). Let $V(\mathscr{L})=\operatorname{Spec}(S(\mathscr{L}))$, the geometric vector bundle associated to the locally free sheaf (line bundle) $\mathscr{L}$ (see [Har77, Exercise 5.18, Page 128]). Then, $V(\mathscr{L})$ is an open subvariety in $\mathbb{P}(\mathscr{E})$ and we have the following commutative diagram

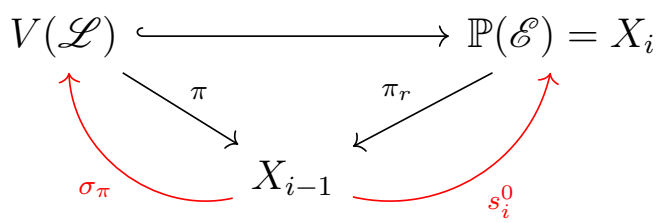

Also note that the section $s_{i}^{0}\left(X_{i-1}\right)$ of $\pi_{i}$ corresponding to the projection $\mathscr{E} \rightarrow \mathcal{O}_{X_{i}}$ is same as the zero section $\sigma_{\pi}\left(X_{i-1}\right)$ of $\pi$. Now consider the following short exact sequence

$$
0 \longrightarrow T_{\pi} \longrightarrow T_{V(\mathscr{L})} \longrightarrow \pi^{*} T_{X_{i-1}} \longrightarrow 0 .
$$

Since the restriction $T_{\left.\right|_{\sigma_{\pi}}\left(X_{i-1}\right)}$ of $T_{\pi}$ to $\sigma_{\pi}\left(X_{r-1}\right)$ is $\check{\mathscr{L}}$, by Lemma 3.7 and by above short exact sequence 3.9 we see that $\mathcal{N}_{\sigma_{\pi}\left(X_{i-1}\right) / V(\mathscr{L})} \simeq \check{\mathscr{L}}$. Hence we conclude that $\mathcal{N}_{X_{i-1} / X_{i}} \simeq \check{\mathscr{L}}$ (here we are identifying $X_{i-1}$ with the section corresponding to the projection $\mathscr{E}=\mathcal{O}_{X_{i-1}} \oplus \mathscr{L} \rightarrow$ $\left.\mathcal{O}_{X_{i-1}}\right)$. This completes the proof of the lemma.

Let $1 \leq i \leq r$. We prove,

\section{Lemma 3.9.}

(1) The toric sections of $\pi_{i}$ are given by $D_{\rho_{i}^{\epsilon}}, \epsilon \in\{+,-\}$. 
(2) The normal bundle $\mathcal{N}_{X_{i-1} / X_{i}}$ of $X_{i-1}$ in $X_{i}$ is given by

$$
\mathcal{N}_{X_{i-1} / X_{i}}=\check{\mathscr{L}_{i-1}}=\mathcal{O}_{X_{i}}\left(D_{\rho_{i}^{+}}\right),
$$

where the line bundle $\mathscr{L}_{i-1}$ is as in the definition of the Bott tower $X_{i}$.

Proof. Proof of (1): Recall that $\pi_{i}$ is a $\mathbb{P}^{1}$-fibration induced by the projection $\bar{\pi}_{i}: \mathbb{Z}^{i} \rightarrow \mathbb{Z}^{i-1}$. For each cone $\sigma \in \Sigma_{F}$ of dimension 1 (which is a maximal cone in $\Sigma_{F}$, where $\Sigma_{F}$ denote the fan of the fiber $\left.\mathbb{P}^{1}\right)$, the subvariety $V(\sigma)$ is an invariant section of $\pi_{i}$, which is an invariant divisor in $X_{i}$. Hence we get two invariant divisors $V\left(\rho_{i}^{+}\right)=D_{\rho_{i}^{+}}$and $V\left(\rho_{i}^{-}\right)=D_{\rho_{i}^{-}}$.

Proof of (2): By Lemma 3.8, we have $\mathcal{N}_{X_{i-1} / X_{i}}=\check{\mathscr{L}}_{i-1}$ and the section $X_{i-1}$ is given by the projection $\mathscr{E}=\mathcal{O}_{X_{i-1}} \oplus \mathscr{L}_{i-1} \rightarrow \mathcal{O}_{X_{i-1}}$. Hence (2) follows from (1).

\section{Primitive Relations of the Bott tower}

4.1. Primitive collections and primitive relations. First recall the notion of primitive collections and primitive relations of a fan $\Sigma$, which are basic tools for the classification of Fano toric varieties due to Batyrev (see [Bat91]).

Definition 4.1. We say $P \subset \Sigma(1)$ is a primitive collection if $P$ is not contained in $\sigma(1)$ for some $\sigma \in \Sigma$ but any proper subset is. Note that if $\Sigma$ is simplicial, primitive collection means that $P$ does not generate a cone in $\Sigma$ but every proper subset does.

Definition 4.2. Let $P=\left\{\rho_{1}, \ldots, \rho_{k}\right\}$ be a primitive collection in a complete simplicial fan $\Sigma$. Recall $u_{\rho}$ is the primitive vector of the ray $\rho \in \Sigma$. Then $\sum_{i=1}^{k} u_{\rho_{i}}$ is in the relative interior of a cone $\gamma_{P}$ in $\Sigma$ with a unique expression

$$
\sum_{i=1}^{k} u_{\rho_{i}}=\sum_{\rho \in \gamma_{P}(1)} c_{\rho} u_{\rho}, c_{\rho} \in \mathbb{Q}_{>0} \text {. Hence we have } \sum_{i=1}^{k} u_{\rho_{i}}-\left(\sum_{\rho \in \gamma_{P}(1)} c_{\rho} u_{\rho}\right)=0 \text {. }
$$

Then we call (4.1) the primitive relation of $X_{\Sigma}$ corresponding to $P$.

Recall that $T \operatorname{Div}\left(X_{\Sigma}\right)$ denote the group of torus-invariant divisors in $X_{\Sigma}$ (see Page 4). Since the fan $\Sigma$ of $X_{r}$ is full dimensional, we have the following short exact sequence

$$
0 \longrightarrow M \stackrel{\varphi_{1}}{\longrightarrow} \operatorname{TDiv}\left(X_{r}\right)=\bigoplus_{\rho \in \Sigma(1)} \mathbb{Z} D_{\rho} \stackrel{\varphi_{2}}{\longrightarrow} \operatorname{Pic}\left(X_{r}\right) \rightarrow 0,
$$

where the maps are given by $\varphi_{1}: m \mapsto \operatorname{div}\left(\chi^{m}\right)$ and $\varphi_{2}: D \mapsto \mathcal{O}_{X_{r}}(D)$ (see [CLS11, Theorem 4.2.1]).

Now we recall some standard notations: Let $X$ be a smooth projective variety, we define

$$
N_{1}(X)_{\mathbb{Z}}:=\left\{\sum_{\text {finite }} a_{i} C_{i}: a_{i} \in \mathbb{Z}, C_{i} \text { irreducible curve in } X\right\} / \equiv
$$

where $\equiv$ is the numerical equivalence, i.e. $Z \equiv Z^{\prime}$ if and only if $D \cdot Z=D \cdot Z^{\prime}$ for all divisors $D$ in $X$. We denote by $[C]$ the class of $C$ in $N_{1}(X)_{\mathbb{Z}}$. Let $N_{1}(X):=N_{1}(X)_{\mathbb{Z}} \otimes \mathbb{R}$. It is a well known fact that $N_{1}(X)$ is a finite dimensional real vector space (see Kle66, Proposition 4, 11 , Chapter IV]). In the case where $X$ is a (smooth projective) toric variety, $N_{1}(X)_{\mathbb{Z}}$ is dual to 
$\operatorname{Pic}(X)$ via the natural pairing (see [CLS11, Proposition 6.3.15]). In our case $X=X_{r}$, there are dual exact sequences:

$$
0 \longrightarrow M \stackrel{\varphi_{1}}{\longrightarrow} \mathbb{Z}^{\Sigma(1)} \stackrel{\varphi_{2}}{\longrightarrow} \operatorname{Pic}\left(X_{r}\right) \longrightarrow 0
$$

and

$$
0 \longrightarrow N_{1}\left(X_{r}\right)_{\mathbb{Z}} \stackrel{\varphi_{2}^{*}}{\longrightarrow} \mathbb{Z}^{\Sigma(1)} \stackrel{\varphi_{1}^{*}}{\longrightarrow} N \longrightarrow 0
$$

where

and

$$
\varphi_{2}^{*}([C])=\left(D_{\rho} \cdot C\right)_{\rho \in \Sigma(1)}, \quad C \text { is an irreducible complete curve in } X_{r}
$$

$$
\varphi_{1}^{*}\left(e_{\rho}\right)=u_{\rho}, \quad e_{\rho} \text { is a standard basis vector of } \mathbb{R}^{\Sigma(1)}
$$

(see [CLS11, Proposition 6.4.1]). Let $P$ be a primitive collection in $\Sigma$. Note that since $X_{r}$ is smooth projective, $P \cap \gamma_{P}(1)=\emptyset$ and

$$
c_{\rho} \in \mathbb{Z}_{>0} \text { for all } \rho \in \gamma_{P}(1)
$$

(see [CLS11, Proposition 7.3.6]). As an element in $\mathbb{Z}^{\sum(1)}$, we write $r(P)=\left(r_{\rho}\right)_{\rho \in \Sigma(1)}$, where

$$
r_{\rho}= \begin{cases}1 & \text { if } \rho \in P \\ -c_{\rho} & \text { if } \rho \in \gamma_{P}(1) \\ 0 & \text { otherwise }\end{cases}
$$

Then by 4.1 we see that

$$
\sum_{\rho \in \sum(1)} r_{\rho} u_{\rho}=0
$$

Hence by the exact sequence (4.3) and by 4.4, we observe that $r(P)$ gives an element in $N_{1}\left(X_{r}\right)_{\mathbb{Z}}$ (see [CLS11, Page 305]). We prove,

Lemma 4.3. Let $P_{i}:=\left\{\rho_{i}^{+}, \rho_{i}^{-}\right\}, 1 \leq i \leq r$. Then $\left\{P_{i}: 1 \leq i \leq r\right\}$ is the set of all primitive collections of the fan $\Sigma$ of $X_{r}$.

Proof. By Theorem 2.6, the cones in the fan $\Sigma$ of $X_{r}$ are generated by subsets of $\left\{e_{1}^{+}, \ldots, e_{r}^{+}, e_{1}^{-}, \ldots, e_{r}^{-}\right\}$and containing no subset of the form $\left\{e_{i}^{+}, e_{i}^{-}\right\}$. Then by Definition 4.1. it is clear that $P_{i}=\left\{\rho_{i}^{+}, \rho_{i}^{-}\right\}$is a primitive collection for all $i$. Also note that again by description of the cones in $\Sigma$, any primitive collection must contain a $P_{i}$ for some $1 \leq i \leq r$.

Fix $1 \leq i \leq r$. Let $Q$ be a collection of one-dimensional cones such that it properly contains $P_{i}$, i.e. there exists $1 \leq j \leq r$ and $j \neq i$ such that $\rho_{j}^{\epsilon} \in Q \supset P_{i}, \epsilon \in\{+,-\}$. Assume that $Q$ is a primitive collection. Then by Definition 4.1, $\left\{\rho_{i}^{+}, \rho_{i}^{-}\right\} \subset Q$ generates a cone in $\Sigma$. This is a contradiction to the description of the cones in $\Sigma$. Therefore, we conclude that $\left\{P_{i}: 1 \leq i \leq r\right\}$ is the set of all primitive collections.

Now we define the Contractible classes from [Cas03]: Let $X$ be a smooth projective toric variety. We define $N E(X)_{\mathbb{Z}}$ in $N_{1}(X)$ by

$$
N E(X)_{\mathbb{Z}}:=\left\{\sum_{\text {finite }} a_{i} C_{i}: a_{i} \in \mathbb{Z}_{\geq 0} \text { and } C_{i} \text { irreducible curve in } X\right\} .
$$

Let $\gamma \in N E(X)_{\mathbb{Z}}$ be primitive (i.e. the generator of $\mathbb{Z}_{\geq 0} \gamma$ ) and such that there exists some irreducible curve in $X$ having numerical class in $\mathbb{Q}_{\geq 0} \gamma$. Then 
Definition 4.4. (see Cas03, Definition 2.3]) The above class $\gamma$ is called contractible if there exists a toric variety $X_{\gamma}$ and an equivariant morphism $\phi_{\gamma}: X \rightarrow X_{\gamma}$, surjective with connected fibers, such that for every irreducible curve $C$ in $X$,

$$
\phi_{\gamma}(C)=\{p t\} \text { if and only if }[C] \in \mathbb{Q}_{\geq 0} \gamma .
$$

Remark 4.5. Note that any contractible class is always a class of some invariant curve and also a primitive relation (see [Cas03, Theorem 2.2] and [Sca09, Page 74]).

Recall the following result from [Cas03, Proposition 3.4].

Proposition 4.6. Let $P=\left\{\rho_{1}, \ldots, \rho_{k}\right\}$ be a primitive collection in $\Sigma$, with the primitive relation $r(P)$ :

$$
\sum_{i=1}^{k} u_{\rho_{i}}-\sum_{\rho \in \gamma_{P}(1)} c_{\rho} u_{\rho}=0 .
$$

Then $r(P)$ is contractible if and only if for every primitive collection $Q$ of $\Sigma$ such that $P \cap Q \neq \emptyset$ and $P \neq Q$, the set $(Q \backslash P) \cup \gamma_{P}(1)$ contains a primitive collection.

4.2. Mori cone. We use the notation as above. Let $X$ be a smooth projective variety. We define $N E(X)$ the real convex cone in $N_{1}(X)$ generated by classes of irreducible curves. The Mori cone $\overline{N E}(X)$ is the closure of $N E(X)$ in $N_{1}(X)$ and it is a strongly convex cone of maximal dimension.

If $X$ is a (smooth projective) toric variety, it is known that $N E(X)_{\mathbb{Z}}$ is generated by the finitely many torus-invariant irreducible curves in $X$ and hence $N E(X)_{\mathbb{Z}}$ is a finitely generated monoid. Hence the cone $N E(X)=\overline{N E}(X)$ is a rational polyhedral cone and we have

$$
\overline{N E}(X)=\sum_{\tau \in \Sigma(r-1)} \mathbb{R}_{\geq 0}[V(\tau)]
$$

where $r=\operatorname{dim}(X)$ and $[V(\tau)] \in N_{1}(X)_{\mathbb{Z}}$ is the class of the toric curve $V(\tau)$. This is called the Toric Cone Theorem (see [CLS11, Theorem 6.3.20]). Let $\tau \in \Sigma(r-1)$ be a wall, that is $\tau=\sigma \cap \sigma^{\prime}$ for some $\sigma, \sigma^{\prime} \in \Sigma(r)$. Let $\sigma$ (respectively, $\sigma^{\prime}$ ) is generated by $\left\{u_{\rho_{1}}, u_{\rho_{2}}, \ldots, u_{\rho_{r}}\right\}$ (respectively, by $\left\{u_{\rho_{2}}, \ldots, u_{\rho_{r+1}}\right\}$ ) and let $\tau$ be generated by $\left\{u_{\rho_{2}}, \ldots, u_{\rho_{r}}\right\}$. Then we get a linear relation,

$$
u_{\rho_{1}}+\sum_{i=2}^{r} b_{i} u_{\rho_{i}}+u_{\rho_{r+1}}=0
$$

The relation (4.6) called wall relation and we have

$$
D_{\rho} \cdot V(\tau)= \begin{cases}b_{i} & \text { if } \rho=\rho_{i} \text { and } i \in\{2,3, \ldots, r\} \\ 1 & \text { if } \rho=\rho_{i} \text { and } i \in\{1, r+1\} \\ 0 & \text { otherwise }\end{cases}
$$

(see CLS11, Proposition 6.4.4 and eq. (6.4.6) page 303]). Now we describe the Mori cone $\overline{N E}\left(X_{r}\right)$ of $X_{r}$ in terms of the primitive relations of $X_{r}$.

Theorem 4.7. $\overline{N E}\left(X_{r}\right)_{\mathbb{Z}}=\sum_{i=1}^{r} \mathbb{Z}_{\geq 0} r\left(P_{i}\right)$. 
Proof. We have

$$
\overline{N E}\left(X_{r}\right)=\sum_{P \in \mathscr{P}} \mathbb{R}_{\geq 0} r(P)
$$

where $\mathscr{P}$ is the set of all primitive collections in $X_{r}$ (see [CLS11, Theorem 6.4.11]). By Lemma 4.3. $\left\{P_{i}: 1 \leq i \leq r\right\}$ is the set of all primitive collections of $X_{r}$. Therefore, we get

$$
\overline{N E}\left(X_{r}\right)=\sum_{i=1}^{r} \mathbb{R}_{\geq 0} r\left(P_{i}\right) \text {. }
$$

By Cas03, Theorem 4.1], we have

$$
\overline{N E}\left(X_{r}\right)_{\mathbb{Z}}=\sum_{\gamma \in \mathscr{C}} \mathbb{Z}_{\geq 0} \gamma
$$

where $\mathscr{C}$ is the set of all contractible classes in $X_{r}$.

By Proposition 4.6, we can see that the primitive relations $r\left(P_{i}\right)$ are contractible classes for $1 \leq i \leq r$. Since any contractible class is a primitive relation, we get

$$
\mathscr{C}=\left\{r\left(P_{i}\right): 1 \leq i \leq r\right\} .
$$

Hence we conclude that

$$
\overline{N E}\left(X_{r}\right)_{\mathbb{Z}}=\sum_{i=1}^{r} \mathbb{Z}_{\geq 0} r\left(P_{i}\right) .
$$

This completes the proof of the theorem.

We have

Corollary 4.8. The set $\left\{r\left(P_{i}\right): 1 \leq i \leq r\right\}$ forms a basis of $N_{1}\left(X_{r}\right)_{\mathbb{Z}}$.

Proof. By Theorem 4.7, $\left\{r\left(P_{i}\right): 1 \leq i \leq r\right\}$ generates the monoid $\overline{N E}\left(X_{r}\right)_{\mathbb{Z}}$ and the cone $\overline{N E}\left(X_{r}\right)$ is of dimension $r$. So $r\left(P_{i}\right)$ for $1 \leq i \leq r$ are linearly independent. Also the group $N_{1}\left(X_{r}\right)_{\mathbb{Z}}$ is generated by $\overline{N E}\left(X_{r}\right)_{\mathbb{Z}}$, hence by $r\left(P_{i}\right)$ for $1 \leq i \leq r$. Hence these form a basis of $N_{1}\left(X_{r}\right)_{\mathbb{Z}}$

Next we describe the primitive relation $r\left(P_{i}\right)$ explicitly by finding the cone $\gamma_{P_{i}}$ in (4.1) for $1 \leq i \leq r$. We also observe that these cones depend on the given matrix corresponding to the Bott tower. We need some notation to state the result. Recall the matrix $M_{r}$ corresponding to the Bott tower $X_{r}$ is

$$
M_{r}=\left[\begin{array}{ccccc}
1 & \beta_{12} & \beta_{13} & \ldots & \beta_{1 r} \\
0 & 1 & \beta_{23} & \ldots & \beta_{2 r} \\
0 & 0 & 1 & \ldots & \beta_{3 r} \\
\vdots & \vdots & & \ddots & \vdots \\
0 & \ldots & \ldots & & 1
\end{array}\right]_{r \times r}
$$

(see Section 2). Fix $1 \leq i \leq r$. Define:

(1) Let $r \geq j>j_{1}=i \geq 1$ and define $a_{1, j}:=\beta_{j_{1} j}$.

(2) Let $r \geq j_{2}>j_{1}$ be the least integer such that $a_{1, j}>0$, then define for $j>j_{2}$

$$
a_{2, j}:=\beta_{i j_{2}} \beta_{j_{2 j}}-\beta_{i j}
$$


(3) Let $k>2$ and let $r \geq j_{k}>j_{k-1}$ be the least integer such that $a_{k-1, j}<0$, then inductively, define for $j>j_{k}$

$$
a_{k, j}:=-a_{k-1, j_{k}} \beta_{j_{k} j}+a_{k-1, j} .
$$

(4) For $j \leq i, b_{j}:=0$, and for $j>i$ define

$$
b_{j}:=a_{l, j} \text { if } j_{l+1} \geq j>j_{l}, l \geq 1 .
$$

Note that we have

$$
b_{j}= \begin{cases}0 & \text { for } j \leq i \\ <0 & \text { for } j \in\left\{j_{3}, \ldots, j_{m}\right\} \\ \geq 0 & \text { otherwise }\end{cases}
$$

(5) Let $I_{i}:=\left\{j_{1}, \ldots, j_{m}\right\}$.

Example 4.9. Let

$$
M_{7}=\left[\begin{array}{ccccccc}
1 & -1 & -1 & -1 & 2 & -1 & 2 \\
0 & 1 & 0 & 2 & -1 & 2 & -1 \\
0 & 0 & 1 & 0 & -1 & -1 & -1 \\
0 & 0 & 0 & 1 & -1 & 2 & -1 \\
0 & 0 & 0 & 0 & 1 & -1 & 2 \\
0 & 0 & 0 & 0 & 0 & 1 & -1 \\
0 & 0 & 0 & 0 & 0 & 0 & 1
\end{array}\right]_{7 \times 7}
$$

Let $i=1$, then $j_{1}=1$ and (1) $a_{1,2}=\beta_{12}=-1$; (2) $a_{1,3}=\beta_{13}=-1$; (3) $a_{1,4}=\beta_{14}=-1$; (4) $a_{1,5}=\beta_{15}=2$; (5) $a_{1,6}=\beta_{16}=-1$; (6) $a_{1,7}=\beta_{17}=2$.

Then $j_{2}=5$ and (1) $a_{2,6}=\beta_{15} \beta_{56}-\beta_{16}=-1$; (2) $a_{2,7}=\beta_{15} \beta_{57}-\beta_{17}=2$.

Then $j_{3}=6$ and $a_{3,7}=-a_{2,6} \beta_{67}+a_{2,7}=-(-1)(-1)+(2)=1$.

Therefore, $I_{1}=\{1,5,6\}$.

Let $1 \leq i \leq r$. Let $\mathscr{A}_{i}:=\left\{e_{j}^{\epsilon_{j}}: 1 \leq j \leq r, b_{j} \neq 0\right.$ and

$$
\epsilon_{j}=\left\{\begin{array}{ll}
+ & \text { for } j \notin I_{i} \\
- & \text { for } j \in I_{i}
\end{array} \quad\right\} .
$$

Remark 4.10. Note that as $b_{j}=0$ for $j \leq i$, we can take $i<j \leq r$ in the definition of $\mathscr{A}_{i}$.

Now we have,

Proposition 4.11. Let $1 \leq i \leq r$. The cone $\gamma_{P_{i}}$ in the primitive relation of $X_{r}$ corresponding to $P_{i}$ is generated by $\mathscr{A}_{i}$.

Before going to the proof we see an example.

Example 4.12. We use same setting as in Example 4.9. By Lemma 4.3, we have $P_{i}=\left\{\rho_{i}^{+}, \rho_{i}^{-}\right\}$ for all $1 \leq i \leq 7$. By definition of $e_{i}^{-}$(see (2.2)), we have

(i) $e_{1}^{-}+e_{1}^{+}=e_{2}^{+}+e_{3}^{+}+e_{4}^{+}-2 e_{5}^{+}+e_{6}^{+}-2 e_{7}^{+}$; (ii) $e_{2}^{-}+e_{2}^{+}=-2 e_{4}^{+}+e_{5}^{+}-2 e_{6}^{+}+e_{7}^{+}$; (iii) $e_{3}^{-}+e_{3}^{+}=e_{5}^{+}+e_{7}^{+}$; (iv) $e_{4}^{-}+e_{4}^{+}=e_{5}^{+}-2 e_{6}^{+}+e_{7}^{+}$; (v) $e_{5}^{-}+e_{5}^{+}=e_{6}^{+}-2 e_{7}^{+}$; (vi) $e_{6}^{-}+e_{6}^{+}=e_{7}^{+}$; (vii) $e_{7}^{-}+e_{7}^{+}=0$. 
Now we describe the cone $\gamma_{P_{1}}$. Observe that in (i) coefficient of $e_{5}^{+}$is negative. By (v), we can see

$$
e_{1}^{-}+e_{1}^{+}=e_{2}^{+}+e_{3}^{+}+e_{4}^{+}+2\left(e_{5}^{-}-e_{6}^{+}+2 e_{7}^{+}\right)+e_{6}^{+}-2 e_{7}^{+}
$$

Then $e_{1}^{-}+e_{1}^{+}=e_{2}^{+}+e_{3}^{+}+e_{4}^{+}+2 e_{5}^{-}-e_{6}^{+}+2 e_{7}^{+}$. By (vi),

$$
e_{1}^{-}+e_{1}^{+}=e_{2}^{+}+e_{3}^{+}+e_{4}^{+}+2 e_{5}^{-}+e_{6}^{-}+e_{7}^{+} \text {. }
$$

In this case, $I_{1}=\{1,5,6\}$ (see Example 4.9) and the cone $\gamma_{P_{1}}$ is generated by

$$
\left\{e_{2}^{+}, e_{3}^{+}, e_{4}^{+}, e_{5}^{-}, e_{6}^{-}, e_{7}^{+}\right\}
$$

Now we prove Proposition 4.11 .

Proof. By 2.2 , for all $1 \leq i \leq r$, we have

$$
e_{i}^{-}+e_{i}^{+}=-\sum_{j>i} \beta_{i j} e_{j}^{+}
$$

If for all $j>i, \beta_{i j} \leq 0$, then the cone $\gamma_{P_{i}}$ is generated by $\left\{e_{j}^{+}: j>i, \beta_{i j}<0\right\}$. If not, choose the least integer $j_{2}>i$ such that $\beta_{i j_{2}}>0$. Now write

$$
e_{i}^{-}+e_{i}^{+}=-\left(\sum_{j_{2}>j>i} \beta_{i j} e_{j}^{+}\right)+\beta_{i j_{2}}\left(-e_{j_{2}}^{+}\right)-\left(\sum_{j>j_{2}} \beta_{i j} e_{j}^{+}\right)
$$

Again by using (4.9), we have

$$
e_{i}^{-}+e_{i}^{+}=-\left(\sum_{j_{2}>j>i} \beta_{i j} e_{j}^{+}\right)+\beta_{i j_{2}}\left(e_{j_{2}}^{-}+\sum_{j>j_{2}} \beta_{j_{2} j} e_{j}^{+}\right)-\left(\sum_{j>j_{2}} \beta_{i j} e_{j}^{+}\right) .
$$

Then

$$
\left.e_{i}^{-}+e_{i}^{+}=-\left(\sum_{j_{2}>j>i} \beta_{i j} e_{j}^{+}\right)+\beta_{i j_{2}} e_{j_{2}}^{-}+\sum_{j>j_{2}}\left(\beta_{i j_{2}} \beta_{j_{2} j}-\beta_{i j}\right) e_{j}^{+}\right)
$$

By definition $a_{2, j}=\beta_{i j_{2}} \beta_{j_{2} j}-\beta_{i j}$, then we have

$$
e_{i}^{-}+e_{i}^{+}=-\left(\sum_{j_{2}>j>i} \beta_{i j} e_{j}^{+}\right)+\beta_{i j_{2}} e_{j_{2}}^{-}+\left(\sum_{j>j_{2}} a_{2, j} e_{j}^{+}\right) .
$$

If $a_{2, j} \geq 0$ for all $j>j_{2}$, then $\gamma_{P_{i}}$ is generated by

$$
\left\{e_{j}^{\epsilon_{j}}: j>i, \epsilon_{j}=+\forall j \neq j_{2} \text {, and } \epsilon_{j}=- \text { for } j=j_{2}\right\} .
$$

Otherwise, choose the least integer $j_{3}>j_{2}$ such that $a_{2, j_{3}}<0$. By substituting $-e_{j_{3}}^{+}$from 4.9 , we get

$$
e_{i}^{-}+e_{i}^{+}=-\left(\sum_{j_{2}>j>i} a_{1, j} e_{j}^{+}\right)+\beta_{i j_{2}} e_{j_{2}}^{-}+\left(\sum_{j_{3}>j>j_{2}} a_{2, j} e_{j}^{+}\right)-a_{2, j_{3}}\left(e_{j_{3}}^{-}+\sum_{j>j_{3}} \beta_{j_{3} j} e_{j}^{+}\right)+\left(\sum_{j>j_{3}} a_{2, j} e_{j}^{+}\right) .
$$

Then,

$$
\left.e_{i}^{-}+e_{i}^{+}=-\left(\sum_{j_{2}>j>i} a_{1, j} e_{j}^{+}\right)+\beta_{i j_{2}} e_{j_{2}}^{-}+\left(\sum_{j_{3}>j>j_{2}} a_{2, j} e_{j}^{+}\right)-a_{2, j_{3}} e_{j_{3}}^{-}+\sum_{j>j_{3}}\left(-a_{2, j_{3}} \beta_{j_{3} j}+a_{2, j}\right) e_{j}^{+}\right) .
$$

By definition $a_{3, j}=-a_{2, j_{3}} \beta_{j j_{3}}+a_{2, j}$, then we have

$$
e_{i}^{-}+e_{i}^{+}=-\left(\sum_{j_{2}>j>i} a_{1, j} e_{j}^{+}\right)+2 e_{j_{2}}^{-}+\left(\sum_{j_{3}>j>j_{2}} a_{2, j} e_{j}^{+}\right)-a_{2, j_{3}} e_{j_{3}}^{-}+\left(\sum_{j>j_{3}} a_{3, j} e_{j}^{+}\right) .
$$


By repeating this process, we get the cone $\gamma_{P_{i}}$ as we required.

Let $1 \leq i \leq r$. Recall $I_{i}=\left\{i=j_{1}, \ldots, j_{m}\right\}$ as in page 13 . Define for $1 \leq j \leq r$,

$$
c_{j}:= \begin{cases}-b_{j} & \text { if } j \in I_{i} \backslash\left\{j_{1}, j_{2}\right\} \\ b_{j} & \text { otherwise }\end{cases}
$$

Set $\gamma_{P_{i}}(1):=\left\{\gamma_{1}, \ldots, \gamma_{l}\right\}$. Then we have

Corollary 4.13. For $1 \leq i \leq r$, the primitive relation $r\left(P_{i}\right)\left(=\left(r_{\rho}\right)_{\rho \in \Sigma(1)}\right)$ of $X_{r}$ given by

$$
r_{\rho}= \begin{cases}1 & \text { for } \rho=\rho_{i}^{+} \text {or } \rho_{i}^{-} \\ -c_{j} & \text { for } \rho=\gamma_{j} \in \gamma_{P_{i}}(1) \\ 0 & \text { otherwise }\end{cases}
$$

Example 4.14. We use Example 4.12. The following can be seen easily from 4.8.).

(1) $\gamma_{P_{1}}(1)=\left\{\rho_{2}^{+}, \rho_{3}^{+}, \rho_{4}^{+}, \rho_{5}^{-}, \rho_{6}^{-}, \rho_{7}^{+}\right\}$.

(2) The primitive relation $r\left(P_{1}\right)=\left(r_{\rho}\right)_{\rho \in \Sigma(1)}$ is given by

$$
r_{\rho}= \begin{cases}1 & \text { for } \rho=\rho_{1}^{+} \text {or } \rho_{1}^{-} \\ -1 & \text { for } \rho=\rho_{k}^{+}, k \in\{2,3,4,7\} \text { and } \rho=\rho_{6}^{-} \\ -2 & \text { for } \rho=\rho_{5}^{-} \\ 0 & \text { otherwise }\end{cases}
$$

Now we describe the primitive relations $r\left(P_{i}\right)$ in terms of intersection of two maximal cones in the fan of $X_{r}$. Let $1 \leq i \leq r$. Let $\mathscr{C}_{i}^{\prime}:=\left\{e_{j}^{\epsilon_{j}}: 1 \leq j \leq r\right.$ and

$$
\epsilon_{j}=\left\{\begin{array}{ll}
+ & \text { if } j \notin I_{i} \backslash\left\{j_{1}\right\} \\
- & \text { if } j \in I_{i}
\end{array}\right\} .
$$

Let $\mathscr{C}_{i}^{\prime \prime}:=\left\{e_{j}^{\epsilon_{j}}: 1 \leq j \leq r\right.$ and

$$
\epsilon_{j}=\left\{\begin{array}{ll}
+ & \text { if } j \notin I_{i} \\
- & \text { if } j \in I_{i}
\end{array}\right\} .
$$

Example 4.15. We use Example 4.12, for $i=1$, we have $I_{1}=\{1,5,6\}$. Then

$$
\mathscr{C}_{1}^{\prime}=\left\{e_{1}^{+}, e_{2}^{+}, e_{3}^{+}, e_{4}^{+}, e_{5}^{-}, e_{6}^{-}, e_{7}^{+}\right\} \text {and } \mathscr{C}_{1}^{\prime \prime}=\left\{e_{1}^{-}, e_{2}^{+}, e_{3}^{+}, e_{4}^{+}, e_{5}^{-}, e_{6}^{-}, e_{7}^{+}\right\} .
$$

We prove the following by using wall relation (see page 12).

Proposition 4.16. Fix $1 \leq i \leq r$. The class of curve $r\left(P_{i}\right)$ is given by

$$
r\left(P_{i}\right)=\left[V\left(\tau_{i}\right)\right],
$$

where $\tau_{i}=\sigma \cap \sigma^{\prime}$ and $\sigma$ (respectively, $\left.\sigma^{\prime}\right)$ is the cone generated by $\mathscr{C}_{i}^{\prime}$ (respectively, by $\left.\mathscr{C}_{i}^{\prime \prime}\right)$. 
Proof. From Corollary 4.13 , we have the following.

$$
e_{i}^{+}+e_{i}^{-}-\sum_{j>i} c_{j} e_{j}^{\epsilon_{j}}=0
$$

where $\epsilon_{j}$ is as in Proposition 4.11. First we show that the set $Q:=\left\{\rho \in \Sigma(1): D_{\rho} \cdot V\left(\tau_{i}\right)>0\right\}$ is not contained in $\sigma(1)$ for any $\sigma \in \Sigma$ (we adapt the arguments of [CLS11, Proof of Theorem 6.4.11, page 306], here we are not assuming the curve $V\left(\tau_{i}\right)$ is extremal). Indeed, suppose $Q \subseteq \sigma(1)$ for some $\sigma \in \Sigma$. Let $D$ be an ample divisor in $X_{r}$ (such exists as $X_{r}$ is projective). Then, we can assume that $D$ is of the form

$$
D=\sum_{\rho \in \Sigma(1)} a_{\rho} D_{\rho}, a_{\rho}=0 \text { for all } \rho \in \sigma(1) \text { and } a_{\rho} \geq 0 \text { for all } \rho \notin \sigma(1)
$$

(see [CLS11, (6.4.10), page 306]). Then we can see

$$
D \cdot V\left(\tau_{i}\right)=\sum_{\rho \notin \sigma(1)} a_{\rho} D_{\rho} \cdot V\left(\tau_{i}\right)
$$

As $Q \subseteq \sigma(1)$, by definition of $Q, D_{\rho} \cdot V\left(\tau_{i}\right) \leq 0$ for $\rho \notin \sigma(1)$. Since $a_{\rho} \geq 0$ for $\rho \notin \sigma(1)$, we get $D \cdot V\left(\tau_{i}\right) \leq 0$, which is a contradiction as $D$ is ample. Therefore, $Q$ is not contained in $\sigma(1)$ for any $\sigma \in \Sigma$. Hence to prove the proposition it is enough to prove

$$
P_{i}=Q\left(:=\left\{\rho \in \Sigma(1): D_{\rho} \cdot V\left(\tau_{i}\right)>0\right\}\right)
$$

(see again [CLS11, Proof of Theorem 6.4.11, page 306]). From (4.10) and by using wall relation, we can see that

$$
D_{\rho} \cdot V\left(\tau_{i}\right)= \begin{cases}1 & \text { if } \rho=\rho_{i}^{+} \text {or } \rho_{i}^{-} \\ -c_{j} & \text { if } \rho=\rho_{j}^{\epsilon_{j}} \text { and } j \in I_{i} \backslash\left\{j_{1}\right\} \\ 0 & \text { otherwise. }\end{cases}
$$

Since $c_{j}$ 's are all positive integers (see (4.4), by Lemma 4.3 we conclude that

$$
P_{i}=\left\{\rho \in \Sigma(1): D_{\rho} \cdot V\left(\tau_{i}\right)>0\right\}
$$

and hence $r\left(P_{i}\right)=\left[V\left(\tau_{i}\right)\right]$. This completes the proof of the proposition.

Example 4.17. In Example 4.12, the curve $r\left(P_{1}\right)=\left[V\left(\tau_{1}\right)\right]$ with $\tau_{1}=\sigma \cap \sigma^{\prime}$ where $\sigma$ is the cone generated by

$$
\mathscr{C}_{1}^{\prime}=\left\{e_{1}^{+}, e_{2}^{+}, e_{3}^{+}, e_{4}^{+}, e_{5}^{-}, e_{6}^{-}, e_{7}^{+}\right\}
$$

and $\sigma^{\prime}$ is the cone generated by

$$
\mathscr{C}_{1}^{\prime \prime}=\left\{e_{1}^{-}, e_{2}^{+}, e_{3}^{+}, e_{4}^{+}, e_{5}^{-}, e_{6}^{-}, e_{7}^{+}\right\} .
$$

Corollary 4.18. $\overline{N E}\left(X_{r}\right)_{\mathbb{Z}}=\sum_{i=1}^{r} \mathbb{Z}_{\geq 0}\left[V\left(\tau_{i}\right)\right]$, where $\tau_{i}$ is as in Proposition 4.16 .

Proof. This follows from Theorem 4.7 and Proposition 4.16 


\section{Ample And nef line BUndles on the Bott tower}

Let $X$ be a smooth projective variety. Recall $N^{1}(X)$ is the real finite dimensional vector space of numerical classes of real divisors in $X$ (see [Kle66, $\S 1$, Chapter IV]). In $N^{1}(X)$, we define the nef cone $N e f(X)$ to be the cone generated by classes of numerically effective divisors and it is a strongly convex closed cone in $N^{1}(X)$. The ample cone $A m p(X)$ of $X$ is the cone in $N^{1}(X)$ generated by classes of ample divisors. Note that the ample cone $A m p(X)$ is interior of the nef cone $N$ ef $(X)$ (see [Kle66, Theorem 1, $\S 2$, Chapter IV]). Recall that the nef cone $N e f(X)$ and the Mori cone $\overline{N E}(X)$ are closed convex cones and are dual to each other (see [Kle66, §2, Chapter IV] ) .

In our case, we have $\operatorname{Pic}\left(X_{r}\right)_{\mathbb{R}}=N^{1}\left(X_{r}\right)$, as the numerical equivalence and linear equivalence coincide (see [CLS11, Proposition 6.3.15]).

In this section, we characterize the ampleness and numerically effectiveness of line bundles on $X_{r}$ and we study the generators of the nef cone of $X_{r}$. We use the notation as in Section 4 . Let $D=\sum a_{\rho} D_{\rho}$ be a toric divisor in $X_{r}$ and for $1 \leq i \leq r$, define

$$
d_{i}:=\left(a_{\rho_{i}^{+}}+a_{\rho_{i}^{-}}-\sum_{\gamma_{j} \in \gamma_{P_{i}}(1)} c_{j} a_{\gamma_{j}}\right) .
$$

Then we prove,

\section{Lemma 5.1.}

(1) The divisor $D$ is ample if and only if $d_{i}>0$ for all $1 \leq i \leq r$.

(2) The divisor $D$ is numerically effective (nef) if and only if $d_{i} \geq 0$ for all $1 \leq i \leq r$.

Proof. Proof of (2): Recall that the primitive relation $r\left(P_{i}\right)$ is given by

$$
r\left(P_{i}\right)=\left(r_{\rho}\right)_{\rho \in \Sigma(1)}
$$

(see page 11). First observe that we have the following

$$
D \cdot r\left(P_{i}\right)=\sum_{\rho \in \Sigma(1)} a_{\rho}\left(D_{\rho} \cdot r\left(P_{i}\right)\right)=\sum_{\rho \in \Sigma(1)} a_{\rho} r_{\rho}
$$

(see [CLS11, Proposition 6.4.1, page 299]). Then by (4.5), we get

$$
D \cdot r\left(P_{i}\right)=\sum_{\rho \in P_{i}} a_{\rho}-\sum_{\rho \in \gamma_{P_{i}}(1)} r_{\rho} a_{\rho}
$$

By Lemma 4.3, we have $P_{i}=\left\{\rho_{i}^{+}, \rho_{i}^{-}\right\}$. Then by Corollary 4.13 , we get

$$
D \cdot r\left(P_{i}\right)=\left(a_{\rho_{i}^{+}}+a_{\rho_{i}^{-}}-\sum_{\gamma_{j} \in \gamma_{P_{i}}(1)} c_{j} a_{\gamma_{j}}\right)=: d_{i} .
$$

Since the nef cone $N e f\left(X_{r}\right)$ and the Mori cone $\overline{N E}\left(X_{r}\right)$ are dual to each other, the divisor $D$ is nef if and only if $D \cdot C \geq 0$ for all torus-invariant irreducible curves $C$ in $X_{r}$. By Theorem 4.7, we have

$$
\overline{N E}\left(X_{r}\right)=\sum_{i=1}^{r} \mathbb{R}_{\geq 0} r\left(P_{i}\right)
$$


Hence $D$ is nef if and only if $D \cdot r\left(P_{i}\right) \geq 0$ for all $1 \leq i \leq r$. Therefore, by (5.1), we conclude that the divisor $D$ is nef if and only if $d_{i} \geq 0$ for all $1 \leq i \leq r$. This completes the proof of (2).

Proof of (1): Recall that the divisor $D$ is ample if and only if its class in $\operatorname{Pic}\left(X_{r}\right)_{\mathbb{R}}$ lies in the interior of the nef cone $N e f\left(X_{r}\right)$. Hence by using similar arguments as in the proof of (2) and the toric Kleiman criterion for ampleness [CLS11, Theorem 6.3.13], we can see that $D$ is ample if and only if $d_{i}>0$ for all $1 \leq i \leq r$.

Next we describe the generators of the nef cone $N e f\left(X_{r}\right)$ of $X_{r}$.

Example 5.2. Let $M_{2}=\left[\begin{array}{cc}1 & -1 \\ 0 & 1\end{array}\right]_{2 \times 2}$. Then $X_{2}=\mathbb{P}\left(\mathcal{O}_{\mathbb{P}^{1}} \oplus \mathcal{O}_{\mathbb{P}^{1}}(1)\right)$, the Hirzebruch surface $\mathscr{H}_{1}$ and the rays $\rho_{1}^{+}, \rho_{1}^{-}, \rho_{2}^{+}$and $\rho_{2}^{-}$of the fan (shown below) of $X_{2}$ are generated by $e_{1}^{+}, e_{1}^{-}=$ $-e_{1}^{+}+e_{2}^{+}, e_{2}^{+}$and $-e_{2}^{+}$respectively.

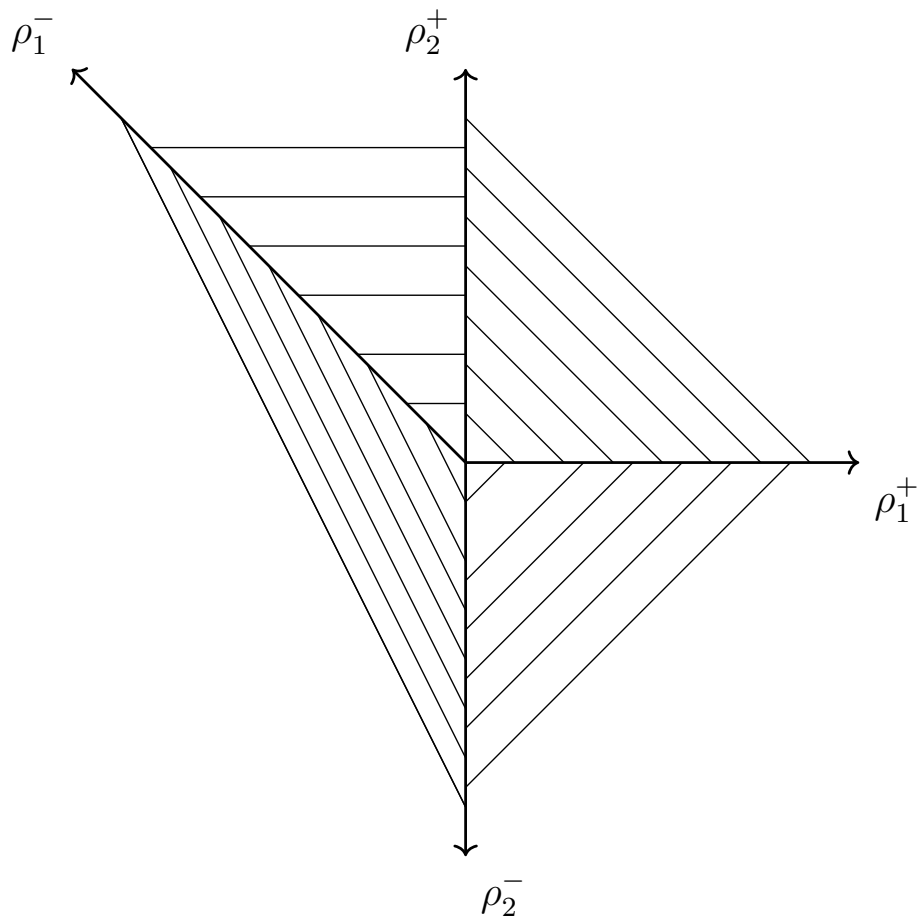

Figure. Fan of Hirzebruck surface $\mathscr{H}_{1}$.

The primitive relations $r\left(P_{1}\right)$ and $r\left(P_{2}\right)$ are given by

$$
r\left(P_{1}\right): e_{1}^{+}+e_{1}^{-}=e_{2} \text { and } r\left(P_{2}\right): e_{2}^{+}+e_{2}^{-}=0 .
$$

By wall relation, we observe that

(1) $D_{\rho_{1}^{+}} \cdot r\left(P_{1}\right)=1$ and $D_{\rho_{1}^{+}} \cdot r\left(P_{2}\right)=0$.

(2) $D_{\rho_{2}^{-}} \cdot r\left(P_{1}\right)=0$ and $D_{\rho_{2}^{-}} \cdot r\left(P_{2}\right)=1$.

Then the dual basis of $\left\{r\left(P_{1}\right), r\left(P_{2}\right)\right\}$ is $\left\{D_{\rho_{1}^{+}}, D_{\rho_{2}^{-}}\right\}$. Hence the generators of the nef cone Nef $\left(\mathscr{H}_{1}\right)$ are $D_{\rho_{1}^{+}}$and $D_{\rho_{2}^{-}}$. Note that by Lemma 3.1. Pic $\left(\mathscr{H}_{1}\right)$ is generated by $\left\{D_{\rho_{1}^{+}}, D_{\rho_{2}^{-}}\right\}$. 
Let $D=a D_{\rho_{1}^{+}}+b D_{\rho_{2}^{-}} \in \operatorname{Pic}\left(\mathscr{H}_{1}\right)$. Then

$$
D \text { is ample if and only if } a>0 \text { and } b>0
$$

(this gives back [CLS11, Example (6.1.16), page 273]).

Now we prove the similar results for $X_{r}$. For $1 \leq m \leq r$, define

$$
J_{m}:=\left\{1 \leq i<m:\left\{\rho_{m}^{+}\right\} \cap \gamma_{P_{i}}(1) \neq \emptyset\right\} .
$$

Remark 5.3. Note that the set $J_{m}$ is the collection of indices $i<m$ for which $u_{\rho_{m}^{+}}$appear in the $\gamma_{P_{i}}$ part of the expression (4.1) for the primitive relation $r\left(P_{i}\right)$.

We set $D_{1}:=D_{\rho_{1}^{+}}$, and for $m>1$ define inductively

$$
D_{m}:= \begin{cases}D_{\rho_{m}^{+}} & \text {if } J_{m}=\emptyset \\ \left(\sum_{k \in J_{m}} c_{\rho_{m}^{+}}^{\gamma_{P_{k}}} D_{k}\right)+D_{\rho_{m}^{+}} & \text {if } J_{m} \neq \emptyset,\end{cases}
$$

where $-c_{\rho_{m}^{+}}^{\gamma_{P_{k}}}$ is the coefficient of $e_{m}^{+}$in the primitive relation $r\left(P_{k}\right)$.

Example 5.4. In Example 5.2 $D_{1}=D_{\rho_{1}^{+}}, J_{2}=\{1\}$ and $D_{2}=D_{1}+D_{\rho_{2}^{+}}$. By using (2.2), we see that $0 \sim \operatorname{div}\left(\chi^{e_{1}^{+}}\right) \sim D_{\rho_{1}^{+}}-D_{\rho_{1}^{-}}$and $0 \sim \operatorname{div}\left(\chi^{e_{2}^{+}}\right) \sim D_{\rho_{2}^{+}}-D_{\rho_{2}^{-}}+D_{\rho_{1}^{-}}$. Hence $D_{2}=$ $D_{1}+D_{\rho_{2}^{+}}=D_{\rho_{2}^{-}}$.

Example 5.5. In Example 4.12,

(1) Recall by (4.8), we have $e_{1}^{-}+e_{1}^{+}=e_{2}^{+}+e_{3}^{+}+e_{4}^{+}+2 e_{5}^{-}+e_{6}^{-}+e_{7}^{+}$. Then, $\gamma_{P_{1}}(1)=\left\{\rho_{2}^{+}, \rho_{3}^{+}, \rho_{4}^{+}, \rho_{5}^{-}, \rho_{6}^{-}, \rho_{7}^{+}\right\}$.

(2) $\gamma_{P_{2}}(1)=\left\{\rho_{4}^{-}, \rho_{5}^{-}, \rho_{6}^{+}, \rho_{7}^{+}\right\} \quad\left(\right.$ since $\left.e_{2}^{+}+e_{2}^{-}=2 e_{4}^{-}+e_{5}^{-}+e_{6}^{+}+e_{7}^{+}\right)$.

(3) $\gamma_{P_{3}}(1)=\left\{\rho_{5}^{+}, \rho_{7}^{+}\right\} \quad$ (since $\left.e_{3}^{+}+e_{3}^{-}=e_{5}^{+}+e_{7}^{+}\right)$

(4) $\gamma_{P_{4}}(1)=\left\{\rho_{5}^{+}, \rho_{6}^{-}, \rho_{7}^{-}\right\} \quad$ (since $\left.e_{4}^{+}+e_{4}^{-}=e_{5}^{+}+2 e_{6}^{-}+e_{7}^{-}\right)$.

(5) $\gamma_{P_{5}}(1)=\left\{\rho_{6}^{+}, \rho_{7}^{-}\right\} \quad$ (since $\left.e_{5}^{+}+e_{5}^{-}=e_{6}^{+}+2 e_{7}^{-}\right)$.

(6) $\gamma_{P_{6}}(1)=\left\{\rho_{7}^{+}\right\} \quad$ (since $\left.e_{6}^{+}+e_{6}^{-}=e_{7}^{+}\right)$.

(7) $\gamma_{P_{7}}(1)=\emptyset . \quad$ (since $\left.e_{7}^{+}+e_{7}^{-}=0\right)$.

Then,

(1) If $m=1$, then $D_{1}=D_{\rho_{1}^{+}}$.

(2) If $m=2$, then $J_{2}=\{1\}$ and $c_{\rho_{2}^{+}}^{\gamma_{P_{1}}}=1$. Hence $D_{2}=D_{1}+D_{\rho_{2}^{+}}$.

(3) If $m=3$, then $J_{3}=\{1\}$ and $c_{\rho_{3}^{+}}^{\gamma_{P_{1}}}=1$. Hence $D_{3}=D_{1}+D_{\rho_{3}^{+}}$.

(4) If $m=4$, then $J_{4}=\{1\}$ and $c_{\rho_{4}^{+}}^{\gamma_{P_{1}}}=1$. Hence $D_{4}=D_{1}+D_{\rho_{4}^{+}}$. 
(5) If $m=5$, then $J_{5}=\{3,4\}$ and $c_{\rho_{5}^{+}}^{\gamma_{P_{3}}}=1 ; c_{\rho_{5}^{+}}^{\gamma_{P_{4}}}=1$. Hence

$$
D_{5}=D_{3}+D_{4}+D_{\rho_{5}^{+}} \text {. }
$$

(6) If $m=6$, then $J_{6}=\{2,5\}$ and $c_{\rho_{6}^{+}}^{\gamma_{P_{2}}}=1 ; c_{\rho_{6}^{+}}^{\gamma_{P_{5}}}=1$. Hence

$$
D_{6}=D_{2}+D_{5}+D_{\rho_{6}^{+}} \text {. }
$$

(7) If $m=7$, then $J_{7}=\{1,2,3,6\}$ and

$$
\begin{gathered}
c_{\rho_{7}^{+}}^{\gamma_{P_{1}}}=1 ; c_{\rho_{7}^{+}}^{\gamma_{P_{2}}}=1 ; c_{\rho_{7}^{+}}^{\gamma_{P_{3}}}=1 ; \text { and } c_{\rho_{7}^{+}}^{\gamma_{P_{6}}}=1 . \text { Hence } \\
D_{7}=D_{1}+D_{2}+D_{3}+D_{6}+D_{\rho_{7}^{+}}
\end{gathered}
$$

We prove,

Proposition 5.6. The set $\left\{D_{i}: 1 \leq i \leq r\right\}$ is dual basis of $\left\{r\left(P_{i}\right): 1 \leq i \leq r\right\}$.

Proof. Fix $1 \leq i \leq r$. By Proposition 4.16, the class of curve corresponding to the primitive relation $r\left(P_{i}\right)$ is given by

$$
r\left(P_{i}\right)=\left[V\left(\tau_{i}\right)\right]
$$

(where $\tau_{i}$ is described as in Proposition 4.16). From Corollary 4.13, the primitive relation $r\left(P_{i}\right)\left(=\left[V\left(\tau_{i}\right)\right]\right)$ is

$$
e_{i}^{+}+e_{i}^{-}-\sum_{j>i} c_{j} e_{j}^{\epsilon_{j}}=0
$$

where $\epsilon_{j}$ is as in Proposition 4.16. Note that this is the wall relation for the torus-invariant curve $V\left(\tau_{i}\right)$. We prove

$$
D_{m} \cdot r\left(P_{i}\right)=D_{m} \cdot V\left(\tau_{i}\right)= \begin{cases}1 & \text { if } i=m \\ 0 & \text { if } i \neq m\end{cases}
$$

By 5.2 and by wall relation, we have

$$
D_{\rho_{m}^{+}} \cdot V\left(\tau_{i}\right)= \begin{cases}1 & \text { for } m=i \\ 0 & \text { for } m<i \\ -c_{\rho_{m}^{+}}^{\gamma_{P_{i}}} & \text { for } m>i \text { and } i \in J_{m} \\ 0 & \text { for } m>i \text { and } i \notin J_{m}\end{cases}
$$

Hence by definition of $D_{m}$, it is clear that

$$
D_{m} \cdot V\left(\tau_{i}\right)= \begin{cases}1 & \text { for } m=i \\ 0 & \text { for } m<i\end{cases}
$$

Now we claim $D_{m} \cdot V\left(\tau_{i}\right)=0$ for all $m>i$. Assume that $m>i$ and write $m=i+j$, where $1 \leq j \leq r-i$. We prove the claim by induction on $j$. If $j=1$, then $D_{m}=D_{i+1}$.

Case 1: If $J_{i+1}=\emptyset$, then $D_{i+1}=D_{\rho_{i+1}^{+}}$. By (5.4), we see that $D_{i+1} \cdot V\left(\tau_{i}\right)=0$.

Case 2: Assume that $J_{i+1} \neq \emptyset$.

Subcase 1: If $i \notin J_{i+1}$, then by (5.4) and (5.5), we can see that $D_{i+1} \cdot V\left(\tau_{i}\right)=0$. 
Subcase 2: If $i \in J_{i+1}$, then by 5.5 , we have $D_{i+1} \cdot V\left(\tau_{i}\right)=c_{\rho_{i+1}^{+}}^{\gamma_{P_{i}}}+\left(D_{\rho_{i+1}^{+}} \cdot V\left(\tau_{i}\right)\right)$.

By 5.4$), D_{\rho_{i+1}^{+}} \cdot V\left(\tau_{i}\right)=-c_{\rho_{i+1}^{+}}^{\gamma_{P_{i}}}$ and hence $D_{i+1} \cdot V\left(\tau_{i}\right)=0$. This proves the claim for $j=1$.

Now assume that $j>1$.

Case 1: If $J_{m}=\emptyset$, then by (5.4) and (5.5), we see that $D_{m} \cdot V\left(\tau_{i}\right)=0$.

Case 2: Assume that $J_{m} \neq \emptyset$.

Subcase 1: If $i \notin J_{m}$, then by (5.4) and (5.5), we can see that

$$
D_{m} \cdot V\left(\tau_{i}\right)=\left(\left(\sum_{k \in J_{m}, k>i} c_{\rho_{m}^{+}}^{\gamma_{P_{k}}} D_{k}\right) \cdot V\left(\tau_{i}\right)\right)+\left(D_{\rho_{m}^{+}} \cdot V\left(\tau_{i}\right)\right) .
$$

By induction on $j, D_{k} \cdot V\left(\tau_{i}\right)=0$ for all $i<k<m$. By (5.4), as $m>i$ and $m \notin J_{m}$, we have $D_{\rho_{m}^{+}} \cdot V\left(\tau_{i}\right)=0$. Hence we conclude that $D_{m} \cdot V\left(\tau_{i}\right)=0$. This completes the proof of the proposition.

We have,

\section{Theorem 5.7.}

(1) The nef cone $N$ ef $\left(X_{r}\right)$ of $X_{r}$ is generated by $\left\{D_{i}: 1 \leq i \leq r\right\}$.

(2) The divisor $D=\sum_{i} a_{i} D_{i}$ is ample if and only if $a_{i}>0$ for all $1 \leq i \leq r$.

Proof. Since the nef cone $N e f\left(X_{r}\right)$ is dual of the Mori cone $\overline{N E}\left(X_{r}\right),(1)$ follows from Proposition 5.6.

Proof of (2): This follows from (1) as the ample cone $A m p\left(X_{r}\right)$ is interior of the nef cone $\operatorname{Nef}\left(X_{r}\right)$.

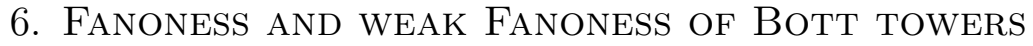

In this section we describe the matrices $M_{r}$ such that the corresponding Bott tower $X_{r}$ is Fano or weak Fano. First recall the Iitaka dimension of a Cartier divisor $D$ in a normal projective variety $X$. Let

$$
N(D):=\left\{m \geq 0: H^{0}(X, \mathscr{L}(m D)) \neq 0\right\},
$$

where $\mathscr{L}(m D)$ is the line bundle associated to $m D$. For $m \in N(D)$, we have a rational map

$$
\phi_{m}: X \rightarrow \mathbb{P}\left(H^{0}(X, \mathscr{L}(m D))^{*}\right) .
$$

If $N(D)$ is empty we define the Iitaka dimension $\kappa(D)$ of $D$ as $-\infty$. Otherwise we define

$$
\kappa(D):=\max _{m \in N(D)}\left\{\operatorname{dim}\left(\phi_{m}(X)\right)\right\} .
$$

Observe that $\kappa(D) \in\{-\infty, 0,1, \ldots, \operatorname{dim}(X)\}$. We say $D$ is $\operatorname{big}$ if $\kappa(D)=\operatorname{dim}(X)$ (see [Laz04, Section 2.2, page 139]). Note that an ample divisor is big.

Lemma 6.1. Let $X$ be a smooth projective variety, let $U$ be an open affine subset of $X$. Let $D$ be an effective divisor with support $X \backslash U$. Then $D$ is big. 
Proof. It suffices to show that there exists an effective divisor $E$ with support $X \backslash U$ such that $E$ is $b i g$. Indeed, we then have $m D=E+F$ for some $m \geq 0$ and for some effective divisor $F$. Then $E+F$ is big and hence so is $D$.

There exists $f_{1}, \ldots, f_{n} \in \mathcal{O}_{X}(U)$ algebraically independent over $\mathbb{C}$, where $n=\operatorname{dim}(X)$. View $f_{1}, \ldots, f_{n}$ as rational functions on $X$, then $f_{1}, \ldots, f_{n} \in H^{0}\left(X, \mathcal{O}_{X}(E)\right)$ for some effective divisor $E$ with support $X \backslash U$ (since $\operatorname{div}\left(f_{i}\right)$ is an effective divisor with support in $X \backslash U$ for $1 \leq i \leq r)$. Thus, the monomials in $f_{1}, \ldots, f_{n}$ of any degree $m$ are linearly independent elements of $H^{0}\left(X, \mathcal{O}_{X}(m E)\right)$. So $\operatorname{dim}\left(H^{0}\left(X, \mathcal{O}_{X}(m E)\right)\right)$ grows like $m^{n}$ as $m \rightarrow \infty$. Hence $E$ is big (see [Laz04, Corollary 2.1.38 and Lemma 2.2.3]) and this completes the proof.

We get the following as a variant of Lemma 6.1.

Corollary 6.2. Let $X$ be a smooth projective variety and $D$ be an effective divisor. Let $\operatorname{supp}(D)$ denotes the support of $D$. If $X \backslash \operatorname{supp}(D)$ is affine, then $D$ is big.

A smooth projective variety $X$ is called Fano (respectively, weak Fano) if its anti-canonical line bundle $-K_{X}$ is ample (respectively, nef and big). To describe our results we use the notation and terminology from Section 1 (see page 2). We prove,

\section{Theorem 6.3.}

(1) $X_{r}$ is Fano if and only if it satisfies I.

(2) $X_{r}$ is weak Fano if and only if it satisfies II.

Proof. Proof of (2): We have

$$
K_{X_{r}}=-\sum_{\rho \in \Sigma(1)} D_{\rho}
$$

(see [CLS11, Theorem 8.2.3] or [Ful93, Page 74]). The anti-canonical line bundle of any projective toric variety is big, since we have

$$
\operatorname{supp}\left(-K_{X_{r}}\right)=X_{r} \backslash\left(\mathbb{C}^{*}\right)^{r},
$$

$\left(\mathbb{C}^{*}\right)^{r}$ is an affine open subset of $X_{r}$, by Corollary 6.2, $-K_{X_{r}}$ is big.

By using Lemma 5.1, we prove that $-K_{X_{r}}$ is nef if and only if $X_{r}$ satisfies $I I$.

Let $D=-K_{X_{r}}$. By (6.1) and by definition of $d_{i}$ for $D$ (see Lemma 5.1), we have

$$
d_{i}=2-\sum_{\gamma_{j} \in \gamma_{P_{i}}(1)} c_{j} .
$$

Then by Lemma 5.1(2), $-K_{X_{r}}$ is nef if and only if $\sum_{\gamma_{j} \in \gamma_{P_{i}}(1)} c_{j} \leq 2$ for all $1 \leq i \leq r$.

First assume that $-K_{X_{r}}$ is nef. Fix $1 \leq i \leq r$. By above discussion, we have

$$
\sum_{\gamma_{j} \in \gamma_{P_{i}}(1)} c_{j} \leq 2 .
$$

Since $c_{j}$ 's are positive integers (see 4.4 ), we get the following situation:

$$
\left|\gamma_{P_{i}}(1)\right|=0 \text { or }\left|\gamma_{P_{i}}(1)\right|=1 \text {, or }\left|\gamma_{P_{i}}(1)\right|=2 \text {. }
$$


Case 1: If $\left|\gamma_{P_{i}}(1)\right|=0$, then by definition of $\gamma_{P_{i}}$ (see Definition 4.2), we have

$$
r\left(P_{i}\right): e_{i}^{+}+e_{i}^{-}=0 .
$$

Then $\left|\eta_{i}^{+}\right|=0=\left|\eta_{i}^{-}\right|$. Hence we see $X_{r}$ satisfies the condition $N_{i}^{1}$.

Case 2: If $\left|\gamma_{P_{i}}(1)\right|=1$, then there exists a unique $r \geq j>i$, such that $\gamma_{j} \in \gamma_{P_{i}}(1)$ and the primitive relation is either

$$
r\left(P_{i}\right): e_{i}^{+}+e_{i}^{-}=c_{j} e_{j}^{+}
$$

or

$$
r\left(P_{i}\right): e_{i}^{+}+e_{i}^{-}=c_{j} e_{j}^{-}
$$

By 6.2 , we get $c_{j}=1$ or 2 .

Subcase (i): Assume that $c_{j}=1$. If the primitive relation is $(6.3)$, then we can see that $\mid \eta_{i}^{\overline{+} \mid=0 \text { and }} c_{j}=-\beta_{i j}=1$. Then $\beta_{i j}=-1$ and hence $X_{r}$ satisfies the condition $N_{i}^{1}$.

If the primitive relation is (6.4), then by $2.2\left|\eta_{i}^{-}\right|=0$ and $\left|\eta_{i}^{+}\right|=1$. Hence $c_{j}=\beta_{i j}=1$ and $\beta_{j k}=0$ for all $k>j$.

Subcase (ii): Assume that $c_{j}=2$. If the primitive relation $r\left(P_{i}\right)$ is $(6.3)$, then $\left|\eta_{i}^{+}\right|=0$ and $\left|\eta_{i}^{-}\right|=1$. So by $(2.2)$, we have $c_{j}=-\beta_{i j}$. If the primitive relation $r\left(P_{i}\right)$ is $(6.4)$, then $\left|\eta_{i}^{+}\right|=1$, $\left|\eta_{i}^{-}\right|=0$ and $\beta_{j k}=0$ for all $k>j$. Again by (2.2), we have $c_{j}=\beta_{i j}$ Thus,

$$
\text { either } \beta_{i j}=-2 \text { or } \beta_{i j}=2 \text {. }
$$

Hence $X_{r}$ satisfies the condition $N_{i}^{2}$.

Case 3: If $\left|\gamma_{P_{i}}(1)\right|=2$, then there exists $r \geq s_{1}>s_{2}>i$ with $\gamma_{s_{1}}, \gamma_{s_{2}} \in \gamma_{P_{i}}(1)$ such that the primitive relation $r\left(P_{i}\right)$ is

$$
r\left(P_{i}\right): e_{i}^{+}+e_{i}^{-}=c_{s_{1}} e_{s_{1}}^{ \pm}+c_{s_{2}} e_{s_{2}}^{ \pm}
$$

Subcase (i): If the primitive relation is $r\left(P_{i}\right):: e_{i}^{+}+e_{i}^{-}=c_{s_{1}} e_{s_{1}}^{+}+c_{s_{2}} e_{s_{2}}^{+}$, by 2.2 we see $\overline{\left|\eta_{i}^{+}\right|=0 \text { and }}\left|\eta_{i}^{-}\right|=2$. By (6.2) and (4.4) ( $c_{i}^{\prime}$ 's are positive integers), we get

$$
c_{s_{1}}=1, c_{s_{2}}=1 \text { and } \beta_{i s_{1}}=\beta_{i s_{2}}=-1 .
$$

Hence $X_{r}$ satisfies the condition $N_{i}^{2}$.

Subcase (ii): If the primitive relation is $r\left(P_{i}\right): e_{i}^{+}+e_{i}^{-}=c_{s_{1}} e_{s_{1}}^{+}+c_{s_{2}} e_{s_{2}}^{-}$, by 2.2 we see $\left|\eta_{i}^{\overline{+}}\right|=1=\left|\eta_{i}^{-}\right|$. Then $\beta_{i s_{1}}=-1, \beta_{i s_{2}}=1$ and $\beta_{s_{2} k}=0$ for all $k>s_{2}$.

Subcase (iii): If the primitive relation is $r\left(P_{i}\right): e_{i}^{+}+e_{i}^{-}=c_{s_{1}} e_{s_{1}}^{-}+c_{s_{2}} e_{s_{2}}^{+}$, by (2.2) we see $\left|\eta_{i}^{+}\right|=1$ and $\beta_{i s_{1}}=1$. Then $\beta_{s_{1} s_{2}}-\beta_{i s_{2}}=1$ and $\beta_{s_{1} k}-\beta_{i k}=0$ for all $k>s_{2}$.

Subcase (iv): If the primitive relation is $r\left(P_{i}\right): e_{i}^{+}+e_{i}^{-}=c_{s_{1}} e_{s_{1}}^{-}+c_{s_{2}} e_{s_{2}}^{-}$, by (2.2) we see $\left|\eta_{i}^{+}\right|=1$ and $\beta_{i s_{1}}=1$. Then $\beta_{s_{1} s_{2}}-\beta_{i s_{2}}=-1$ and $\beta_{i s_{2}}-\beta_{s_{1} s_{2}}-\beta_{s_{2} k}=0$ for all $k>s_{2}$.

Hence $X_{r}$ satisfies the condition $N_{i}^{2}$. Therefore, we conclude that if $X_{r}$ is weak Fano then $X_{r}$ satisfies the condition $I I$. Similarly, we can prove by using Lemma 5.1(2), if $X_{r}$ satisfies $I I$ then $X_{r}$ is weak Fano. This completes the proof of (2).

Proof of (1): This follows by using similar arguments as in the proof of (2) and Lemma $5.1(1)$. 
6.1. Local rigidity of Bott towers. Now we prove some vanishing results for the cohomology of tangent bundle of the Bott tower $X_{r}$ and we get some local rigidity results. Let $T_{X_{r}}$ denotes the tangent bundle of $X_{r}$. Then we have

Corollary 6.4. If $X_{\tilde{w}}$ satisfies $I$, then $H^{i}\left(X_{\tilde{w}}, T_{X_{\tilde{w}}}\right)=0$ for all $i \geq 1$.

Proof. If $X_{r}$ satisfies $I$, then by Theorem 6.3, $X_{r}$ is Fano variety. By [BB96, Proposition 4.2], since $X_{r}$ is a smooth Fano toric variety, we get $H^{i}\left(X_{r}, T_{X_{r}}\right)=0$ for all $i \geq 1$.

It is well known that by Kodaira-Spencer theory, the vanishing of $H^{1}\left(X, T_{X}\right)$ implies that $X$ is locally rigid, i.e. admits no local deformations (see Huy06, Proposition 6.2.10, page 272]). Then by above result we have

Corollary 6.5. The Bott tower $X_{r}$ is locally rigid if it satisfies $I$.

\section{Log FAnOness of BotT TOWERS}

Recall that a pair $(X, D)$ of a normal projective variety $X$ and an effective $\mathbb{Q}$-divisor $D$ is Kawamata log terminal (klt) if $K_{X}+D$ is $\mathbb{Q}$-Cartier, and for all proper birational maps $f: Y \longrightarrow X$, the pull back $f^{*}\left(K_{X}+D\right)=K_{Y}+D^{\prime}$ satisfies $f_{*} K_{Y}=K_{X}$ and $\left\lfloor D^{\prime}\right\rfloor \leq 0$, where $\left\lfloor\sum_{i} a_{i} D_{i}\right\rfloor=\sum_{i}\left\lfloor a_{i}\right\rfloor D_{i},\lfloor x\rfloor$ is the greatest integer $\leq x$. The pair $(X, D)$ is called log Fano if it is klt and $-\left(K_{X}+D\right)$ is ample.

We recall here, a condition for the anti-canonical line bundle to be big (see [CG13]). Let $X$ be a $\mathbb{Q}$ - Gorenstein projective normal variety over $\mathbb{C}$. If $X$ admits a divisor $D$ with the pair $(X, D)$ being log Fano then $-K_{X}$ is big (In CG13 there is a necessary and sufficient condition that $X$ is log Fano (or "Fano type") variety, see [CG13, Theorem 1.1] for more details on this ) .

If $X$ is smooth and $D$ is a normal crossing divisor, the pair $(X, D)$ is log Fano if and only if $\lfloor D\rfloor=0$ and $-\left(K_{X}+D\right)$ is ample (see [KM08, Lemma 2.30, Corollary 2.31 and Definition 2.34]). In case of toric variety $X$ see also [CLS11, Definition 11.4.23 and Proposition 11.4.24, page 558]. We use notation as in Lemma 5.1. Let $D=\sum_{\rho \in \Sigma(1)} a_{\rho} D_{\rho}$ be a toric divisor in $X_{r}$, with $a_{\rho}^{\prime} s$ in $\mathbb{Q}_{\geq 0}$ and $\lfloor D\rfloor=0$. For $1 \leq i \leq r$, define

$$
k_{i}:=d_{i}-2+\sum_{\gamma_{j} \in \gamma_{P_{i}}(1)} c_{j} .
$$

Then we prove,

Theorem 7.1. The pair $\left(X_{r}, D\right)$ is log Fano if and only if $k_{i}<0$ for all $1 \leq i \leq r$.

Proof. From the above discussion by the condition on $D$, the pair $\left(X_{r}, D\right)$ is $\log$ Fano if and only if $-\left(K_{X_{r}}+D\right)$ is ample. Note that as $-K_{X_{r}}=\sum_{\rho \in \Sigma(1)} D_{\rho}$, we get

$$
-\left(K_{X_{r}}+D\right)=\sum_{\rho \in \Sigma(1)}\left(1-a_{\rho}\right) D_{\rho} .
$$

By Lemma 5.1. $-\left(K_{X_{r}}+D\right)$ is ample if and only if

$$
\left(\left(1-a_{\rho_{i}^{+}}\right)+\left(1-a_{\rho_{i}^{-}}\right)-\sum_{\gamma_{j} \in \gamma_{P_{i}}(1)} c_{j}\left(1-a_{\gamma_{j}}\right)\right)>0 \text { for all } 1 \leq i \leq r .
$$


Recall the definition of $d_{i}$ for $D$,

$$
d_{i}=a_{\rho_{i}^{+}}+a_{\rho_{i}^{-}}-\sum_{\gamma_{j} \in \gamma_{P_{i}}(1)} c_{j} a_{\gamma_{j}}
$$

Then we have

$$
\left(\left(1-a_{\rho_{i}^{+}}\right)+\left(1-a_{\rho_{i}^{-}}\right)-\sum_{\gamma_{j} \in \gamma_{P_{i}}(1)} c_{j}\left(1-a_{\gamma_{j}}\right)\right)=-\left(d_{i}-2+\sum_{\gamma_{j} \in \gamma_{P_{i}}(1)} c_{j}\right) .
$$

Hence in (7.1)

$$
\left(\left(1-a_{\rho_{i}^{+}}\right)+\left(1-a_{\rho_{i}^{-}}\right)-\sum_{\gamma_{j} \in \gamma_{P_{i}}(1)} c_{j}\left(1-a_{\gamma_{j}}\right)\right)=-k_{i} \text { for all } 1 \leq i \leq r
$$

and we conclude that $-\left(K_{X_{r}}+D\right)$ is ample if and only if $k_{i}<0$ for all $1 \leq i \leq r$. This completes the proof of the theorem.

\section{Extremal RAys and Mori Rays of the Bott TOWER}

In this section we study the extremal rays and Mori rays of Mori cone of $X_{r}$. First we recall some definitions. Let $V$ be a finite dimensional vector space over $\mathbb{R}$ and let $K$ be a (closed) cone in $V$. A subcone $Q$ in $K$ is called extremal if $u, v \in K, u+v \in Q$ then $u, v \in Q$. A face of $K$ is an extremal subcone. A one-dimensional face is called an extremal ray. Note that an extremal ray is contained in the boundary of $K$.

Let $X$ be a smooth projective variety. An extremal ray $R$ in $\overline{N E}(X) \subset N_{1}(X)$ is called Mori if $R \cdot K_{X}<0$, where $K_{X}$ is the canonical divisor in $X$. Recall that $\overline{N E}\left(X_{r}\right)$ is a strongly convex rational polyhedral cone of maximal dimension in $N_{1}\left(X_{r}\right)$. We prove,

\section{Theorem 8.1.}

(1) The class of curves $r\left(P_{i}\right)$ for $1 \leq i \leq r$ are all extremal rays in the Mori cone $\overline{N E}\left(X_{r}\right)$ of $X_{r}$.

(2) Fix $1 \leq i \leq r$, the class of curve $r\left(P_{i}\right)$ is Mori ray if and only if either $\left|\gamma_{P_{i}}(1)\right|=0$, or $\left|\gamma_{P_{i}}(1)\right|=1$ with $c_{j}=1$ for $\gamma_{j} \in \gamma_{P_{i}}(1)$.

Proof. Proof of (1): This follows from Theorem 4.7 and Corollary 4.8.

Proof of (2): By (1), $r\left(P_{i}\right) 1 \leq i \leq r$ are all extremal rays in $\overline{N E}\left(X_{r}\right)$. Hence for $1 \leq i \leq r$, $r\left(P_{i}\right)$ is Mori if $K_{X r} \cdot r\left(P_{i}\right)<0$. Since $K_{X_{r}}=-\sum_{\rho \in \Sigma(1)} D_{\rho}$, we can see by Corollary 4.13 and by similar arguments as in the proof of Lemma 5.1 .

$$
K_{X_{r}} \cdot r\left(P_{i}\right)=-2+\sum_{\gamma_{j} \in \gamma_{P_{i}}(1)} c_{j} .
$$

Thus if $K_{X r} \cdot r\left(P_{i}\right)<0$, then

$$
\sum_{\gamma_{j} \in \gamma_{P_{i}}(1)} c_{j}<2
$$

As $c_{j}$ are all positive integers (see (4.4)), we get either $\left|\gamma_{P_{i}}(1)\right|=0$, or $\left|\gamma_{P_{i}}(1)\right|=1$ and $c_{j}=1$ for $\gamma_{j} \in \gamma_{P_{i}}(1)$. Similarly, by using (8.1) we can prove the converse. This completes the proof of the theorem. 
Acknowledgements: I would like to thank Michel Brion for valuable discussions, many critical comments and for encouragement throughout the preparation of this article.

\section{REFERENCES}

[AS14] D. Anderson and A. Stapledon, Schubert varieties are log Fano over the integers, Proceedings of the American Mathematical Society 142 (2014), no. 2, 409-411.

[Bat91] V. V. Batyrev, On the classification of smooth projective toric varieties, Tohoku Mathematical Journal, Second Series 43 (1991), no. 4, 569-585.

[BB96] F. Bien and M. Brion, Automorphisms and local rigidity of regular varieties, Compositio Mathematica 104 (1996), no. 1, 1-26 (eng).

[BS58] R. Bott and H. Samelson, Applications of the theory of Morse to symmetric spaces, American Journal of Mathematics (1958), 964-1029.

[Cas03] C. Casagrande, Contractible classes in toric varieties, Mathematische Zeitschrift 243 (2003), no. 1, 99-126.

[CG13] P. Cascini and Y. Gongyo, On the anti-canonical ring and varieties of Fano type, Saitama Math. J 30 (2013), 27-38.

[Cha17a] B.N. Chary, On Fano and weak Fano Bott-Samelson-Demazure-Hansen varieties, to appear in Journal of Pure and Applied Algebra, https://doi.org/10.1016/j.jpaa.2017.10.006.

[Cha17b] _ A note on toric degeneration of a Bott-Samelson-Demazure-Hansen variety, in preparation.

[CK17] B.N. Chary and S.S. Kannan, Rigidity of a Bott-Samelson-Demazure-Hansen variety for PSp $(2 n, \mathbb{C})$, Journal of Lie Theory 27 (2017), 435-468.

[CKP] B.N. Chary, S.S. Kannan, and A.J. Parameswaran, Automorphism group of a Bott-Samelson-DemazureHansen variety for non reduced case, in preparation.

[CKP15] _ _ Automorphism group of a Bott-Samelson-Demazure-Hansen variety, Transformation Groups 20 (2015), no. 3, 665-698.

[CMS10] S. Choi, M. Masuda, and D. Suh, Topological classification of generalized Bott towers, Transactions of the American Mathematical Society 362 (2010), no. 2, 1097-1112.

[CS11] S. Choi and D. Y. Suh, Properties of Bott manifolds and cohomological rigidity, Algebraic \& Geometric Topology 11 (2011), no. 2, 1053-1076.

[Civ05] Y. Civan, Bott towers, crosspolytopes and torus actions, Geometriae Dedicata 113 (2005), no. 1, 55-74.

[CR05] Y. Civan and N. Ray, Homotopy decompositions and k-theory of Bott towers, K-theory 34 (2005), no. 1, $1-33$.

[CLS11] D.A. Cox, J.B. Little, and H.K. Schenck, Toric varieties, Graduate studies in mathematics, American Mathematical Soc., 2011.

[DJ91] M. W. Davis and T. Januszkiewicz, Convex polytopes, Coxeter orbifolds and torus actions, Duke Math. J 62 (1991), no. 2, 417-451.

[Ful93] W. Fulton, Introduction to toric varieties, no. 131, Princeton University Press, 1993.

[GK94] M. Grossberg, Y. Karshon, Bott towers, complete integrability, and the extended character of representations, Duke Mathematical Journal 76 (1994), no. 1, 23-58.

[Har70] R. Hartshorne, Ample subvarieties of algebraic varieties, Lecture Notes in Mathematics, vol. 156, Springer, 1970.

[Har77] _ _ Algebraic geometry, vol. 52, Springer Science \& Business Media, 1977.

[Huy06] D. Huybrechts, Complex geometry: an introduction, Springer Science \& Business Media, 2006.

[Ish12] H. Ishida, Filtered cohomological rigidity of Bott towers, Osaka Journal of Mathematics 49 (2012), no. 2, $515-522$.

[Kle66] S.L Kleiman, Toward a numerical theory of ampleness, Annals of Mathematics (1966), 293-344.

[Kle80] _ Relative duality for quasi-coherent sheaves, Compositio Mathematica 41 (1980), no. 1, 39-60.

[KM08] J. Kollár and S. Mori, Birational geometry of algebraic varieties, vol. 134, Cambridge university press, 2008.

[Laz04] R.K. Lazarsfeld, Positivity in algebraic geometry I: Classical setting: line bundles and linear series, vol. 48, Springer Science \& Business Media, 2004.

[Mil16] J. S. Milne, Etale cohomology (PMS-33), vol. 33, Princeton university press, 2016. 
[Oda88] T. Oda, Convex bodies and algebraic geometry- An introduction to the theory of toric varieties, vol. (3)15, Springer-Verlag, Berlin Heidelberg NewYork, 1988.

[PK16] A.J. Parameswaran and P. Karuppuchamy, Toric degeneration of Bott-Samelson-Demazure-Hansen varieties, arXiv preprint arXiv:1604.01998 (2016).

[Sca09] A. Scaramuzza, Algorithms for projectivity and extremal classes of a smooth toric variety, Experimental Mathematics 18 (2009), no. 1, 71-84.

[VT15] B. M. Victor and P.E. Taras, Toric topology, vol. 204, Providence: American Mathematical Society, 2015.

B. Narasimha Chary, Institut Fourier, UMr 5582 du CNRS, Université de Grenoble Alpes, CS 40700, 38058, Grenoble Cedex 09, France., Email: narasimha-chary.bonala@univ-GrenobleAPLES.FR 